\title{
STRUCTURAL CONVERGENCE RESULTS FOR APPROXIMATION OF DOMINANT SUBSPACES FROM BLOCK KRYLOV SPACES*
}

\author{
PETROS DRINEAS ${ }^{\dagger}$, ILSE C. F. IPSEN I $^{\ddagger}$ EUGENIA-MARIA KONTOPOULOU§ ${ }^{\S}$ AND \\ MALIK MAGDON-ISMAIL $\uparrow$
}

\begin{abstract}
This paper is concerned with approximating the dominant left singular vector space of a real matrix $\mathbf{A}$ of arbitrary dimension, from block Krylov spaces generated by the matrix $\mathbf{A A}^{T}$ and the block vector AX. Two classes of results are presented. First are bounds on the distance, in the two and Frobenius norms, between the Krylov space and the target space. The distance is expressed in terms of principal angles. Second are quality of approximation bounds, relative to the best approximation in the Frobenius norm. For starting guesses $\mathbf{X}$ of full column-rank, the bounds depend on the tangent of the principal angles between $\mathbf{X}$ and the dominant right singular vector space of $\mathbf{A}$. The results presented here form the structural foundation for the analysis of randomized Krylov space methods. The innovative feature is a combination of traditional Lanczos convergence analysis with optimal approximations via least squares problems.
\end{abstract}

Key words. Singular value decomposition, least squares, principal angles, gap-amplifying polynomials, random matrices.

AMS subject classification. 15A18, 15A42, 65F15, 65F20, 68W20.

1. Introduction. Randomized methods for low-rank approximations from Krylov spaces are starting to emerge in the Theoretical Computer Science community [34, 43. This motivated us to produce a "proof of concept" for the approximation of dominant subspaces from Krylov spaces.

Low-rank versus subspace approximations. Our focus is the approximation of a dominant subspace of $\mathbf{A} \in \mathbb{R}^{m \times n}$. This is a different and harder problem than a low-rank approximation of $\mathbf{A}$. To wit, the objective of a low-rank approximation is a matrix $\mathbf{Z}$ with orthonormal columns that makes $\left\|\mathbf{A}-\mathbf{Z} \mathbf{Z}^{T} \mathbf{A}\right\|$ small in some unitarily invariant norm 21, 44. In contrast, a subspace approximation aims at a space $\mathcal{K}$ that has a small angle with the dominant target space, which is the space spanned by the singular vectors associated with the top $k$ left singular vectors of $\mathbf{A}$.

For a dominant subspace to be well-defined, the top $k$ singular values must be separated by a gap from the remaining singular values of $\mathbf{A}$. In contrast, a low-rank approximation can do without a singular value gap. Accuracy results for dominant subspace computations are automatically informative for low-rank approximations,

*The work of the first and third authors was supported in part by NSF grants IIS-1302231 and NSF IIS-1447283. The work of the second author was supported in part by the XDATA Program of the Defense Advanced Research Projects Agency (DARPA), administered through Air Force Research Laboratory contract FA8750-12-C-0323. The work of the fourth author was partially supported by NSF grant IIS 1124827, and by the Army Research Laboratory under Cooperative Agreement Number W911NF-09-2-0053 (the ARL Network Science CTA). The views and conclusions contained in this document are those of the authors and should not be interpreted as representing the official policies, either expressed or implied, of the Army Research Laboratory or the U.S. Government. The U.S. Government is authorized to reproduce and distribute reprints for Government purposes notwithstanding any copyright notation here on.

$\dagger$ Department of Computer Science, Purdue University, West Lafayette, IN, pdrineas@purdue.edu

${ }_{\ddagger}$ Department of Mathematics, North Carolina State University, Raleigh, NC, ipsen@ncsu.edu

$\S$ Department of Computer Science, Purdue University, West Lafayette, IN, ekontopo@purdue.edu

IDepartment of Computer Science, Rensselaer Polytechnic Institute, Troy, NY, magdon@cs.rpi.edu 
but not vice versa. It is in this sense that dominant subspace approximations are harder.

This paper. We consider block Krylov space methods for computing dominant left singular vector spaces of general rectangular matrices and we present structural, deterministic bounds on the quality of the subspaces, for essentially general starting guesses. The innovative feature is a fusion of eigenvalue and singular value technology: We combine a traditional Lanczos convergence analysis [38, with optimal approximations via least squares problems 10, 11.

Our long-term goal is to put randomized Krylov space approximations on a firm numerical footing. However, at this preliminary first step, we make a few idealized assumptions:

1. The block Krylov spaces have maximal dimension.

2. The analysis assumes exact arithmetic and does not address the implementation of numerically stable recursions.

Future work will need to deal with the challenging issues of finite precision arithmetic and viable numerical implementations, including recursions, numerical stability, maintaining orthogonality, deflation, adaptation of block size, and restarting. Empirical evaluations will have to assess whether the bounds are tight enough to be informative in practice.

Overview. We start with a brief summary of our contributions (Section 2), followed by a comparison to existing work (Section 3). Auxiliary results (Section 4) set the stage for the proof of the main Theorems (Sections 15, 6, 7 and Appendix $\mathrm{A}$ ). We end the main part of the paper with a perspective on open problems (Section 8).

2. Results. After setting the context (Section 2.1), we give a brief summary of our bounds for: The distance between the Krylov space and the dominant left singular space (Section 2.2); a particular dominant subspace approximation from the Krylov space (Section 2.3); and the polynomials appearing in the approximation (Section 2.4). We end this section with a discussion of options for bounding the distance between the initial guess and the dominant right singular vector space (Section 2.5).

2.1. Setting. To approximate the dominant left singular vector subspace of a matrix $\mathbf{A} \in \mathbb{R}^{m \times n}$, given a starting guess $\mathbf{X} \in \mathbb{R}^{n \times s}$, we construct the Krylov space in $1 \mathbf{A A}^{T}$ and $\mathbf{A X}$,

$$
\mathcal{K}_{q} \equiv \mathcal{K}_{q}\left(\mathbf{A} \mathbf{A}^{T}, \mathbf{A X}\right)=\operatorname{range}\left(\mathbf{A X} \quad\left(\mathbf{A A}^{T}\right) \mathbf{A X} \quad \cdots \quad\left(\mathbf{A A}^{T}\right)^{q} \mathbf{A X}\right) .
$$

We assume maximal dimension, $\operatorname{dim}\left(\mathcal{K}_{q}\right)=(q+1) s$.

In contrast to [4, [5, the matrix $\mathbf{A}$ occurs not only in the powers $\mathbf{A} \mathbf{A}^{T}$ but also has a direct effect on the starting guess through $\mathbf{A X}$. Furthermore, $\mathbf{X}$ is not required to have orthonormal columns and, at times, not even linearly independent columns.

Let $\mathbf{A}=\mathbf{U} \boldsymbol{\Sigma} \mathbf{V}^{T}$ be the full SVD of $\mathbf{A}$, so that $\boldsymbol{\Sigma} \in \mathbb{R}^{m \times n}$, and $\mathbf{U} \in \mathbb{R}^{m \times m}$ and $\mathbf{V} \in \mathbb{R}^{n \times n}$ are orthogonal matrices. For a positive integer $1 \leq k<\operatorname{rank}(\mathbf{A})$, identify the dominant spaces by partitioning

$$
\boldsymbol{\Sigma}=\left(\begin{array}{cc}
\boldsymbol{\Sigma}_{k} & \\
& \boldsymbol{\Sigma}_{k, \perp}
\end{array}\right), \quad \mathbf{U}=\left(\begin{array}{ll}
\mathbf{U}_{k} & \mathbf{U}_{k, \perp}
\end{array}\right), \quad \mathbf{V}=\left(\begin{array}{ll}
\mathbf{V}_{k} & \mathbf{V}_{k, \perp}
\end{array}\right),
$$

\footnotetext{
${ }^{1}$ The superscript $T$ denotes the transpose, and $\|\cdot\|_{2}$ the two norm.
} 
where the diagonal matrix $\boldsymbol{\Sigma}_{k}$ contains the $k$ largest singular values, hence is nonsingular. For the dominant subspaces to be well-defined, the dominant $k$ singular values of $\mathbf{A}$ must be strictly larger than the remaining ones, $1 /\left\|\boldsymbol{\Sigma}_{k}^{-1}\right\|_{2}>\left\|\boldsymbol{\Sigma}_{k, \perp}\right\|_{2}>0$.

2.2. Krylov space angles. We present bounds for the distance between the Krylov space $\mathcal{K}_{q}$ and the dominant left singular vector space range $\left(\mathbf{U}_{k}\right)$. Theorem 2.1 bounds the distance between $\mathcal{K}_{q}$ and the whole space, while Theorem 2.2 bounds the distance between $\mathcal{K}_{q}$ and an individual left singular vector. The distances are represented in terms of principal angles.

Theorem 2.1 below is in the spirit of Rayleigh-Ritz bounds 9, 18. It indicates how well the Krylov space $\mathcal{K}_{q}$ captures the targeted dominant left singular vector space range $\left(\mathbf{U}_{k}\right)$ in both the two norm and the Frobenius norm. Denote by $\boldsymbol{\Theta}\left(\mathcal{K}_{q}, \mathbf{U}_{k}\right) \in$ $\mathbb{R}^{k \times k}$ the diagonal matrix of principal angles between $\mathcal{K}_{q}$ and range $\left(\mathbf{U}_{k}\right)$, and by $\boldsymbol{\Theta}\left(\mathbf{X}, \mathbf{V}_{k}\right) \in \mathbb{R}^{k \times k}$ the diagonal matrix of the principal angles between range $(\mathbf{X})$ and range $\left(\mathbf{V}_{k}\right)$. Principal angles are discussed in detail in Section 4.3 .

THEOREM 2.1. Let $\phi(x)$ be a polynomial of degree $2 q+1$ with odd powers only, such that $\phi\left(\boldsymbol{\Sigma}_{k}\right)$ is nonsingular. If $\operatorname{rank}\left(\mathbf{V}_{k}^{T} \mathbf{X}\right)=k$, then 2

$$
\left\|\sin \boldsymbol{\Theta}\left(\mathcal{K}_{q}, \mathbf{U}_{k}\right)\right\|_{2, F} \leq\left\|\phi\left(\boldsymbol{\Sigma}_{k, \perp}\right)\right\|_{2}\left\|\phi\left(\boldsymbol{\Sigma}_{k}\right)^{-1}\right\|_{2}\left\|\mathbf{V}_{k, \perp}^{T} \mathbf{X}\left(\mathbf{V}_{k}^{T} \mathbf{X}\right)^{\dagger}\right\|_{2, F} .
$$

If, in addition, $\mathbf{X}$ has orthornomal or linearly independent columns, then

$$
\left\|\mathbf{V}_{k, \perp}^{T} \mathbf{X}\left(\mathbf{V}_{k}^{T} \mathbf{X}\right)^{\dagger}\right\|_{2, F}=\left\|\tan \boldsymbol{\Theta}\left(\mathbf{X}, \mathbf{V}_{k}\right)\right\|_{2, F}
$$

and

$$
\left\|\sin \boldsymbol{\Theta}\left(\mathcal{K}_{q}, \mathbf{U}_{k}\right)\right\|_{2, F} \leq\left\|\phi\left(\boldsymbol{\Sigma}_{k, \perp}\right)\right\|_{2}\left\|\phi\left(\boldsymbol{\Sigma}_{k}\right)^{-1}\right\|_{2}\left\|\tan \boldsymbol{\Theta}\left(\mathbf{X}, \mathbf{V}_{k}\right)\right\|_{2, F}
$$

Proof. See Section $\mathbf{5}$ for general and orthonormal $\mathbf{X}$; and Appendix $\mathbf{A}$ for $\mathbf{X}$ with linearly independent columns.

Theorem 2.1 is reminiscent of the eigenvalue bounds [26, (2.18)] which contain a tangent on the left. The term $\left\|\mathbf{V}_{k, \perp}^{T} \mathbf{X}\left(\mathbf{V}_{k}^{T} \mathbf{X}\right)^{\dagger}\right\|_{2, F}$ already appeared in previous analyses of randomized algorithms [15, 16, 17, 32], and bounds for it are discussed in Section 2.5. If $\mathbf{X}$ is a random starting guess, such as a random sign matrix, a random Gaussian matrix 3 or a matrix with randomly chosen orthonormal columns, then state-of-the-art matrix concentration inequalities can be called upon.

In the special case where $\mathbf{X}$ has linearly independent columns the bounds admit a geometric interpretation: They depend on the tangents of angles between range $(\mathbf{X})$ and the dominant right singular vector space range $\left(\mathbf{V}_{k}\right)$. The full-rank assumption for $\mathbf{V}_{k}^{T} \mathbf{X}$ means that the spaces range $\left(\mathbf{V}_{k}\right)$ and $\operatorname{range}(\mathbf{X})$ are sufficiently close, with all principal angles being less than $\pi / 2$.

Next, Theorem 2.2 bounds the distances between $\mathcal{K}_{q}$ and individual left singular vectors of $\mathbf{A}$. To this end, distinguish the $k$ dominant singular values and associated left singular vectors,

$$
\boldsymbol{\Sigma}_{k}=\operatorname{diag}\left(\begin{array}{lll}
\sigma_{1} & \cdots & \sigma_{k}
\end{array}\right), \quad \mathbf{U}_{k}=\left(\begin{array}{lll}
\mathbf{u}_{1} & \cdots & \mathbf{u}_{k}
\end{array}\right) .
$$

\footnotetext{
${ }^{2}$ The subscript $F$ denotes the Frobenius norm.

${ }^{3}$ The elements of a random Gaussian matrix are independent identically distributed normal random variables with mean zero and variance one.
} 
THEOREm 2.2. Let $\phi(x)$ be a polynomial of degree $2 q+1$ with odd powers only, such that $\phi\left(\boldsymbol{\Sigma}_{k}\right)$ is nonsingular. If $\operatorname{rank}\left(\mathbf{V}_{k}^{T} \mathbf{X}\right)=k$, then

$$
\left|\sin \boldsymbol{\Theta}\left(\mathcal{K}_{q}, \mathbf{u}_{i}\right)\right| \leq \frac{\left\|\phi\left(\boldsymbol{\Sigma}_{k, \perp}\right)\right\|_{2}}{\left|\phi\left(\sigma_{i}\right)\right|}\left\|\mathbf{V}_{k, \perp}^{T} \mathbf{X}\left(\mathbf{V}_{k}^{T} \mathbf{X}\right)^{\dagger}\right\|_{2}, \quad 1 \leq i \leq k .
$$

If, in addition, $\mathbf{X}$ has orthonormal columns, then

$$
\left|\sin \boldsymbol{\Theta}\left(\mathcal{K}_{q}, \mathbf{u}_{i}\right)\right| \leq \frac{\left\|\phi\left(\boldsymbol{\Sigma}_{k, \perp}\right)\right\|_{2}}{\left|\phi\left(\sigma_{i}\right)\right|}\left\|\tan \boldsymbol{\Theta}\left(\mathbf{X}, \mathbf{V}_{k}\right)\right\|_{2}, \quad 1 \leq i \leq k .
$$

Proof. See Section 6. ㅁ

In the special case when $\mathbf{X}$ has orthonormal columns, the angle between a single left singular vector and $\mathcal{K}_{q}$ is bounded by all angles between $\mathbf{X}$ and the right singular vector space range $\left(\mathbf{V}_{k}\right)$.

2.3. Approximations from a Krylov space. The results here are motivated by work in the Theoretical Computer Science community on Randomized Linear Algebra [15. There, a common objective is the best rank- $k$ approximation to $\mathbf{A}$ with respect to a unitarily invariant norm,

$$
\mathbf{A}_{k} \equiv \mathbf{U}_{k} \boldsymbol{\Sigma}_{k} \mathbf{V}_{k}^{T}
$$

The particular approximation $\hat{\mathbf{U}}_{k}$ computed by Proto-Algorithm 2.1 guarantees a strong optimality property in the projection $\hat{\mathbf{U}}_{k} \hat{\mathbf{U}}_{k}^{T} \mathbf{A}$ : It is the best rank- $k$ approximation to $\mathbf{A}$ from $\mathcal{K}_{q}$ with respect to the Frobenius norm (see Lemma 7.1).

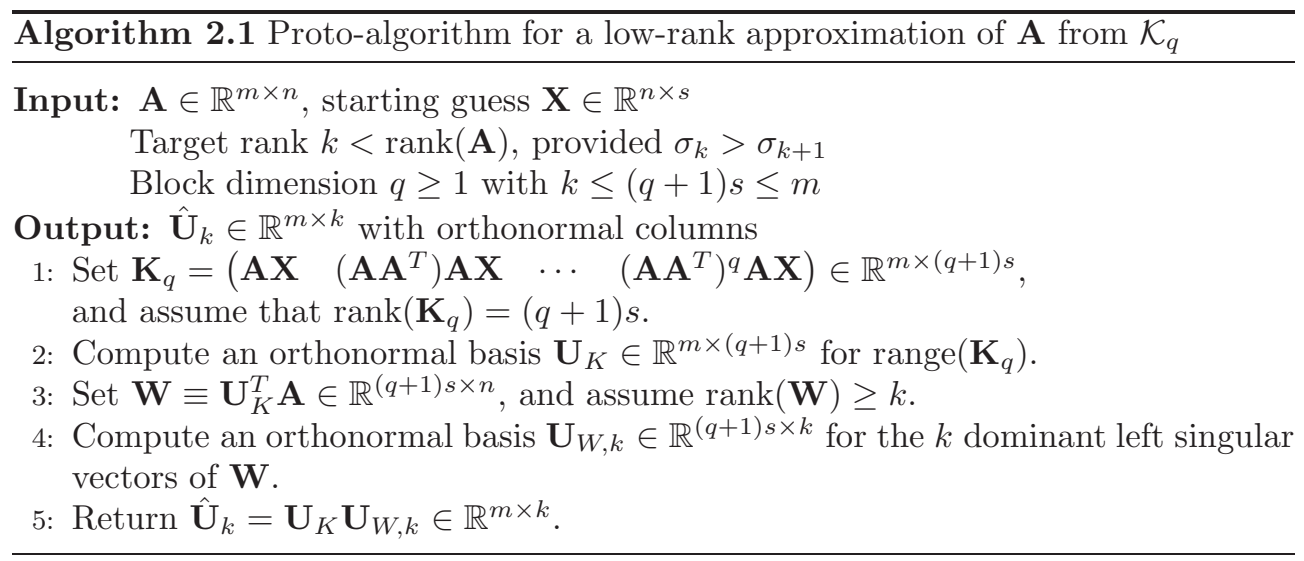

Theorem 2.3 presents a quality-of-approximation result for $\hat{\mathbf{U}}_{k}$. To this end we distinguish the orthonormal columns of $\hat{\mathbf{U}}_{k}=\left(\begin{array}{lll}\hat{\mathbf{u}}_{1} & \ldots & \hat{\mathbf{u}}_{k}\end{array}\right) \in \mathbb{R}^{m \times k}$ and set

$$
\hat{\mathbf{U}}_{i} \equiv\left(\begin{array}{lll}
\hat{\mathbf{u}}_{1} & \ldots & \hat{\mathbf{u}}_{i}
\end{array}\right) \in \mathbb{R}^{m \times i}, \quad 1 \leq i \leq k,
$$

and

$$
\Delta \equiv\left\|\phi\left(\boldsymbol{\Sigma}_{k, \perp}\right)\right\|_{2}\left\|\mathbf{V}_{k, \perp}^{T} \mathbf{X}\left(\mathbf{V}_{k}^{T} \mathbf{X}\right)^{\dagger}\right\|_{F}
$$


THEOREM 2.3. Let $\phi(x)$ be a polynomial of degree $2 q+1$ with odd powers only, such that $\phi\left(\boldsymbol{\Sigma}_{k}\right)$ is nonsingular, and $\phi\left(\sigma_{i}\right) \geq \sigma_{i}$ for $1 \leq i \leq k$. If $\operatorname{rank}\left(\mathbf{V}_{k}^{T} \mathbf{X}\right)=k$, then for $1 \leq i \leq k$,

$$
\begin{aligned}
\left\|\mathbf{A}-\hat{\mathbf{U}}_{i} \hat{\mathbf{U}}_{i}^{T} \mathbf{A}\right\|_{F} & \leq\left\|\mathbf{A}-\mathbf{A}_{i}\right\|_{F}+\Delta \\
\left\|\mathbf{A}-\hat{\mathbf{U}}_{i} \hat{\mathbf{U}}_{i}^{T} \mathbf{A}\right\|_{2} & \leq\left\|\mathbf{A}-\mathbf{A}_{i}\right\|_{2}+\Delta \\
\sigma_{i}-\Delta & \leq\left\|\hat{\mathbf{u}}_{i}^{T} \mathbf{A}\right\|_{2} \leq \sigma_{i} .
\end{aligned}
$$

If, in addition, $\mathbf{X}$ has orthonormal columns, then

$$
\Delta=\left\|\phi\left(\boldsymbol{\Sigma}_{k, \perp}\right)\right\|_{2}\left\|\tan \boldsymbol{\Theta}\left(\mathbf{X}, \mathbf{V}_{k}\right)\right\|_{F} .
$$

\section{Proof. See Section 7 व}

Bounds of the form (2.5) were already proposed in [34, Theorem 1] as a finer, vector-wise, way to capture the quality of approximations to individual left singular vectors of A. Empirical evidence [34] suggests that error metrics of the form (2.3) and (2.4) indicate the quality of the aggregate approximation and are therefore coarser than (2.5).

2.4. Judicious choice of polynomials. We show the existence of and present bounds for the polynomials in Theorems 2.1, 2.2. and 2.3. The strict inequality $\operatorname{rank}(\mathbf{A})>k$ in Algorithm 2.1 allows us to express the relative singular gap as

$$
\frac{\sigma_{k}-\sigma_{k+1}}{\sigma_{k+1}} \geq \gamma>0
$$

which is equivalent to $\sigma_{k} \geq(1+\gamma) \sigma_{k+1}>0$.

Lemma 2.4. If (2.6) holds, then there exists a polynomial $\phi(x)$ of degree $2 q+1$ with odd powers only, such that $\phi\left(\sigma_{i}\right) \geq \sigma_{i}>0$ for $1 \leq i \leq k$, and

$$
\left|\phi\left(\sigma_{i}\right)\right| \leq \frac{4 \sigma_{k+1}}{2^{(2 q+1) \min \{\sqrt{\gamma}, 1\}}}, \quad i \geq k+1 .
$$

Hence

$$
\left\|\phi\left(\boldsymbol{\Sigma}_{k}\right)^{-1}\right\|_{2} \leq \sigma_{k}^{-1} \quad \text { and } \quad\left\|\phi\left(\boldsymbol{\Sigma}_{k, \perp}\right)\right\|_{2} \leq \frac{4 \sigma_{k+1}}{2^{(2 q+1) \min }\{\sqrt{\gamma}, 1\}}
$$

Proof. See Section 4.5, प

We apply Lemma 2.4 to the previous results, first for the special case when $\mathbf{X}$ has linearly independent columns. Abbreviate

$$
\Gamma(\boldsymbol{\Theta}, \gamma, q) \equiv 4 \frac{\left\|\tan \boldsymbol{\Theta}\left(\mathbf{X}, \mathbf{V}_{k}\right)\right\|_{2}}{2^{(2 q+1) \min \{\sqrt{\gamma}, 1\}}} .
$$

To keep things short, we consider only the two-norm bound for Theorem 2.1

Corollary 2.5. Let (2.6) hold and $\operatorname{rank}\left(\mathbf{V}_{k}^{T} \mathbf{X}\right)=k$. If $\mathbf{X}$ has orthonormal columns, then

$$
\left\|\sin \Theta\left(\mathcal{K}_{q}, \mathbf{U}_{k}\right)\right\|_{2} \leq \Gamma(\boldsymbol{\Theta}, \gamma, q) \frac{\sigma_{k+1}}{\sigma_{k}} \leq \frac{\Gamma(\boldsymbol{\Theta}, \gamma, q)}{1+\gamma}
$$


and

$$
\left|\sin \boldsymbol{\Theta}\left(\mathcal{K}_{q}, \mathbf{u}_{i}\right)\right| \leq \Gamma(\boldsymbol{\Theta}, \gamma, q) \frac{\sigma_{k+1}}{\sigma_{i}}, \quad 1 \leq i \leq k .
$$

Proof. Apply Lemma 2.4 to Theorems 2.1 and 2.2 口

Corollary 2.6. Let (2.6) hold and $\operatorname{rank}\left(\mathbf{V}_{k}^{T} \mathbf{X}\right)=k$. If $\mathbf{X}$ has orthonormal columns, then Theorem 2.3 holds with

$$
\Delta \leq \Gamma(\boldsymbol{\Theta}, \gamma, q) \sigma_{k+1},
$$

so that for $1 \leq i \leq k$

$$
\begin{aligned}
\left\|\mathbf{A}-\hat{\mathbf{U}}_{i} \hat{\mathbf{U}}_{i}^{T} \mathbf{A}\right\|_{F} & \leq\left\|\mathbf{A}-\mathbf{A}_{i}\right\|_{F}+\Gamma(\boldsymbol{\Theta}, \gamma, q) \sigma_{k+1}, \\
\left\|\mathbf{A}-\hat{\mathbf{U}}_{i} \hat{\mathbf{U}}_{i}^{T} \mathbf{A}\right\|_{2} & \leq\left\|\mathbf{A}-\mathbf{A}_{i}\right\|_{2}+\Gamma(\boldsymbol{\Theta}, \gamma, q) \sigma_{k+1}, \\
\sigma_{i}-\Gamma(\boldsymbol{\Theta}, \gamma, q) \sigma_{k+1} & \leq\left\|\hat{\mathbf{u}}_{i}^{T} \mathbf{A}\right\|_{2} \leq \sigma_{i} .
\end{aligned}
$$

Proof. Apply Lemma 2.4 to Theorem 2.3 ,

To achieve an additive error of $\Gamma(\boldsymbol{\Theta}, \gamma, q) \leq \epsilon$, set $q$ to be the smallest integer that exceeds

$$
q \geq \frac{1}{2 \min \{\sqrt{\gamma}, 1\}}\left(\log _{2} 4\left\|\tan \boldsymbol{\Theta}\left(\mathbf{X}, \mathbf{V}_{k}\right)\right\|_{2}-\log _{2} \epsilon\right) .
$$

Thus, as the singular value gap $\gamma$ decreases, the dimension of the space $\mathcal{K}_{q}$ increases. More specifically, $q$ increases logarithmically with higher target accuracy $\epsilon$ and increasing distance of $\mathbf{X}$ from the dominant right singular vector space of $\mathbf{A}$.

If $\mathbf{X}$ is rank deficient then Corollaries 2.5 and 2.6 still hold with

$$
\Gamma(\boldsymbol{\Theta}, \gamma, q)=4 \frac{\left\|\mathbf{V}_{k, \perp}^{T} \mathbf{X}\left(\mathbf{V}_{k}^{T} \mathbf{X}\right)^{\dagger}\right\|_{2}}{2^{(2 q+1) \min \{\sqrt{\gamma}, 1\}}} .
$$

2.5. The initial guess. It remains to bound $\left\|\mathbf{V}_{k, \perp}^{T} \mathbf{X}\left(\mathbf{V}_{k}^{T} \mathbf{X}\right)^{\dagger}\right\|_{2, F}$. The simplest way might be strong submultiplicativity,

$$
\left\|\mathbf{V}_{k, \perp}^{T} \mathbf{X}\left(\mathbf{V}_{k}^{T} \mathbf{X}\right)^{\dagger}\right\|_{2, F} \leq\left\|\mathbf{V}_{k, \perp}^{T} \mathbf{X}\right\|_{2, F}\left\|\left(\mathbf{V}_{k}^{T} \mathbf{X}\right)^{\dagger}\right\|_{2}=\frac{\left\|\mathbf{V}_{k, \perp}^{T} \mathbf{X}\right\|_{2, F}}{\sigma_{k}\left(\mathbf{V}_{k}^{T} \mathbf{X}\right)}
$$

followed by separate bounds for the individual factors.

Ideally, the starting guess $\mathbf{X}$ should be close to range $\left(\mathbf{V}_{k}\right)$ and far away from range $\left(\mathbf{V}_{k, \perp}\right)$, so that $\sigma_{k}\left(\mathbf{V}_{k}^{T} \mathbf{X}\right)$ is large and $\left\|\mathbf{V}_{k, \perp}^{T} \mathbf{X}\right\|_{2, F}$ is small. The assumption $\sigma_{k}\left(\mathbf{V}_{k}^{T} \mathbf{X}\right)>0$ is critical for our results, hence a necessary condition for the userspecified matrix $\mathbf{X} \in \mathbb{R}^{n \times s}$ is $\operatorname{rank}(\mathbf{X}) \geq k$, while trying to keep the column dimension $s \geq k$ small.

If $\mathbf{X}$ is a random Gaussian, then $\sigma_{k}\left(\mathbf{V}_{k}^{T} \mathbf{X}\right)$ is bounded away from zero with high probability even for $s=k$. However, there are many other choices for $\mathbf{X}$ that come with lower bounds for $\sigma_{k}\left(\mathbf{V}_{k}^{T} \mathbf{X}\right)$. They include random sign matrices [1, 30], the fast randomized Hadamard transform [2, 39, the subsampled randomized Hadamard transform [17, 42, the fast randomized discrete cosine transform [36, and input sparsity time embeddings $[13,33,35$. 
In contrast, keeping $\left\|\mathbf{V}_{k, \perp}^{T} \mathbf{X}\right\|_{F}$ small is relatively easy. For typical random matrices $\mathbf{X}$, one can show that, with high probability,

$$
\left\|\mathbf{V}_{k, \perp}^{T} \mathbf{X}\right\|_{2, F} \leq c\left\|\mathbf{V}_{k, \perp}\right\|_{F} \leq c \sqrt{n}
$$

where $c$ is a small constant.

For instance, if $s=1$ and $\mathbf{X}$ is a Gaussian column vector, then

$$
\mathbf{E}\left[\left\|\mathbf{V}_{k, \perp}^{T} \mathbf{X}\right\|_{F}^{2}\right]=\left\|\mathbf{V}_{k, \perp}^{T}\right\|_{F}^{2} \leq n .
$$

Markov's inequality guarantees that, with probability at least .9 ,

$$
\left\|\mathbf{V}_{k, \perp}^{T} \mathbf{X}\right\|_{F} \leq \sqrt{10 n}
$$

Essentially all randomized embedding matrices satisfy variants of (2.8), and we expect the iteration count $q$ in (2.7) to be logarithmic in $n$.

From a numerical point of view, a starting guess $\mathbf{X}$ with orthonormal columns is preferable. Thus one could pick a random matrix $\mathbf{X}$ and apply a thin QR decomposition $\mathbf{X}=\mathbf{Q R}$. However, this significantly complicates the derivation of bounds for $\left\|\mathbf{V}_{k, \perp}^{T} \mathbf{X}\right\|_{2, F}$ and $\left\|\left(\mathbf{V}_{k}^{T} \mathbf{X}\right)^{\dagger}\right\|_{2}$, as most matrix concentration inequalities apply only to the original random matrix $\mathbf{X}$, not to its orthonormal basis $\mathbf{Q}$. For instance, if $\mathbf{X}$ is a random matrix whose entries are \pm 1 with equal probability, then $\mathbf{Q}$ does not inherit this property. Fortunately, the subsampled Hadamard transform [17, 42] is one of a few random matrices with orthonormal columns, hence amenable to application of matrix concentration inequalities.

3. Comparison to existing work. Our work on subspace computations is motivated by a recent probabilistic approach for low-rank approximations via block Krylov spaces 34 .

Randomized Methods. Analyses of numerical methods that compute dominant subspaces and eigenvectors from randomized starting vectors date back at least to the 1980s. They include the power method and inverse iteration [14, 24], and information theoretic analyses of Lanczos methods 27, 28,

Current analyses in Theoretical Computer Science focus on low-rank approximations 21, 44, rather than subspace computations, and as such tend not to produce bounds for the accuracy of subspaces such as those in Section 2.2.

A popular approach towards low-rank approximation is subspace iteration, which makes use of only the last iterate $\left(\mathbf{A A}^{T}\right)^{q} \mathbf{A X}[21,44$. Then came block Krylov methods, which exploit all of the iterates $\left(\mathbf{A A}^{T}\right)^{j} \mathbf{A X}, 0 \leq j \leq q$. The analysis in 34 relies on generalized matrix functions [3, 22, but is limited to Gaussian random matrices for starting guesses $\mathbf{X} \in \mathbb{R}^{n \times k}$, and Chebyshev polynomials for $\phi$. The eponymous gap-dependent bound [34, Theorem 13] requires a gap between the $k$ th and $(k+1)$ st singular values, and can be considered a special case of Theorem 2.3 . However, [34, Theorems 10,11, and 12] do not require a singular value gap such as (2.6). Such gap-independent bounds are informative for low-rank approximations, but not for computations of specific subspaces, as explained in Section 1.

Close on the heels of 34 is 43 , with a focus on gap-independent bounds of the type (2.4) and random Gaussian starting guesses [43, Theorem 3.1]. The proof techniques in 43 resemble ours, and leverage our prior work, see Lemma 7.1] and 10, but numerical issues are not addressed. 
Traditional, deterministic methods. Although non-numerical in nature as well, our results are nevertheless guided in spirit by foundational work on eigenvalue and invariant subspace computations, including the standard Lanczos convergence analysis [38, Section 6.6], a geometric view of Krylov space methods [6, 7], block Lanczos methods 29, 37; and Rayleigh-Ritz bounds [9, 18, but also by Krylov space methods for singular value problems [4, 5].

A more detailed comparison, though, seems elusive due to differences in both, the computational problem and the algorithm. The analyses in [6, 7] target vector rather than block methods, for eigenvalues and invariant subspaces of non-Hermitian matrices, with a concern for restarting. The block methods in [29, 37] are Lanczos methods for Hermitian eigenvalue problems, and the analyses exploit the (block) tridiagonal structure resulting from recursions. Although singular value problems are considered in 44 and in [5] with block methods, the Krylov spaces are different and the focus is on algorithmic issues of augmenting and restarting the Lanczos process, rather than subspace distances. Krylov spaces For the solution of ill-posed least squares problems via LSQR, 25] analyzes the accuracy of a regularized solution, by bounding the sine between $\mathcal{K}_{j}\left(\mathbf{A}^{T} \mathbf{A}, \mathbf{A}^{T} \mathbf{v}\right)$ and a dominant right singular vector space; however all singular values must be distinct.

In the context of low-rank approximations, 40] proposed a Lanczos bidiagonalization with one-sided reorthogonalization.

In contrast, the context of this paper is singular vector spaces for general matrices of any dimension; and an algorithm that is not tied to a particular recursion and, due to steps 3-6, is not a straight-forward Krylov method. Furthermore, the key feature of our analyses is a least squares approach [10, 11] that assures the quality of the approximation.

4. Auxiliary results. We review submultiplicative inequalities for norms, the matrix Pythagoras theorem, and solutions of multiple right-hand side least squares problems in the two norm (Section 4.1). We also present expressions for elements of the Krylov space $\mathcal{K}_{q}$ (Section 4.2), review angles between subspaces (Section 4.3), and introduce gap-amplifying polynomials (Section 4.4).

4.1. Norm inequalities, Pythagoras, and least squares. We make frequent use of the strong sub-multiplicativity of the Frobenius norm [23, page 211]. For matrices $\mathbf{Y}_{1} \in \mathbb{R}^{m \times k}$ and $\mathbf{Y}_{2} \in \mathbb{R}^{k \times n}$,

$$
\begin{aligned}
\left\|\mathbf{Y}_{1} \mathbf{Y}_{2}\right\|_{F} & \leq\left\|\mathbf{Y}_{1}\right\|_{2}\left\|\mathbf{Y}_{2}\right\|_{F} \\
\left\|\mathbf{Y}_{1} \mathbf{Y}_{2}\right\|_{F} & \leq\left\|\mathbf{Y}_{1}\right\|_{F}\left\|\mathbf{Y}_{2}\right\|_{2}
\end{aligned}
$$

If, in addition, $\operatorname{rank}\left(\mathbf{Y}_{1}\right)=\operatorname{rank}\left(\mathbf{Y}_{2}\right)$, then [8, Theorem 2.2.3]

$$
\left(\mathbf{Y}_{1} \mathbf{Y}_{2}\right)^{\dagger}=\mathbf{Y}_{2}^{\dagger} \mathbf{Y}_{1}^{\dagger}
$$

Lemma 4.1 (Matrix Pythagoras). Let $\mathbf{A}, \mathbf{B} \in \mathbb{R}^{m \times n}$. If $\mathbf{A}^{T} \mathbf{B}=\mathbf{0}$ then

$$
\|\mathbf{A}+\mathbf{B}\|_{F}^{2}=\|\mathbf{A}\|_{F}^{2}+\|\mathbf{B}\|_{F}^{2} .
$$

Proof. From $\mathbf{A}^{T} \mathbf{B}=\mathbf{0}$ and the linearity of the trace

$$
\begin{aligned}
\|\mathbf{A}+\mathbf{B}\|_{F}^{2} & =\operatorname{trace}\left(\mathbf{A}^{T} \mathbf{A}+\mathbf{A}^{T} \mathbf{B}+\mathbf{B}^{T} \mathbf{A}+\mathbf{B}^{T} \mathbf{B}\right) \\
& =\operatorname{trace}\left(\mathbf{A}^{T} \mathbf{A}\right)+\operatorname{trace}\left(\mathbf{B}^{T} \mathbf{B}\right)=\|\mathbf{A}\|_{F}^{2}+\|\mathbf{B}\|_{F}^{2} .
\end{aligned}
$$


References to optimal solutions for multiple right-hand side least squares problems in the two norm are hard to find. Lemma 4.2 below is easy to prove in the Frobenius norm. An elaborate proof for Schatten $p$ norms can be found in 31, Theorems 3.2 and 3.3]. For the sake of completeness, we present a straightforward proof for the two norm.

Lemma 4.2. Let $\mathbf{A} \in \mathbb{R}^{m \times n}$ and $\mathbf{B} \in \mathbb{R}^{m \times p}$. Then $\mathbf{A}^{\dagger} \mathbf{B}$ is a solution of

$$
\min _{\mathbf{X} \in \mathbb{R}^{n \times p}}\|\mathbf{B}-\mathbf{A X}\|_{2}
$$

with least squares residual $\left\|\left(\mathbf{I}-\mathbf{A A}^{\dagger}\right) \mathbf{B}\right\|_{2}=\min _{\mathbf{X} \in \mathbb{R}^{n \times p}}\|\mathbf{B}-\mathbf{A X}\|_{2}$.

Proof. Let $\mathbf{A}=\mathbf{U} \boldsymbol{\Sigma} \mathbf{V}^{T}$ be a thin SVD, and let $\left(\begin{array}{ll}\mathbf{U} & \mathbf{U}_{\perp}\end{array}\right) \in \mathbb{R}^{m \times m}$ be an orthogonal matrix. Any $\mathbf{X} \in \mathbb{R}^{n \times p}$ satisfies

$$
\begin{aligned}
\|\mathbf{B}-\mathbf{A X}\|_{2}^{2} & =\left\|\mathbf{U U}^{T}(\mathbf{B}-\mathbf{A X})+\mathbf{U}_{\perp} \mathbf{U}_{\perp}^{T}(\mathbf{B}-\mathbf{A X})\right\|_{2}^{2} \\
& =\left\|\mathbf{U}\left(\mathbf{U}^{T} \mathbf{B}-\mathbf{\Sigma} \mathbf{V}^{T} \mathbf{X}\right)+\mathbf{U}_{\perp} \mathbf{U}_{\perp}^{T} \mathbf{B}\right\|_{2}^{2} \\
& =\left\|\mathbf{U} \mathbf{T}_{1}+\mathbf{U}_{\perp} \mathbf{T}_{2}\right\|_{2}^{2},
\end{aligned}
$$

where $\mathbf{T}_{1} \equiv \mathbf{U}^{T} \mathbf{B}-\boldsymbol{\Sigma} \mathbf{V}^{T} \mathbf{X}$ and $\mathbf{T}_{2} \equiv \mathbf{U}_{\perp}^{T} \mathbf{B}$. Let $\mathbf{y}_{\text {opt }} \in \mathbb{R}^{p}$ with $\left\|\mathbf{y}_{\text {opt }}\right\|_{2}=1$ satisfy $\left\|\mathbf{U}_{\perp} \mathbf{T}_{2} \mathbf{y}_{\text {opt }}\right\|_{2}^{2}=\left\|\mathbf{U}_{\perp} \mathbf{T}_{2}\right\|_{2}^{2}$. The vector Pythagoras theorem implies

$$
\begin{aligned}
\|\mathbf{B}-\mathbf{A X}\|_{2}^{2} & \geq\left\|\left(\mathbf{U} \mathbf{T}_{1}+\mathbf{U}_{\perp} \mathbf{T}_{2}\right) \mathbf{y}_{\text {opt }}\right\|_{2}^{2} \\
& =\left\|\mathbf{U} \mathbf{T}_{1} \mathbf{y}_{\text {opt }}\right\|_{2}^{2}+\left\|\mathbf{U}_{\perp} \mathbf{T}_{2} \mathbf{y}_{\text {opt }}\right\|_{2}^{2} \geq\left\|\mathbf{U}_{\perp} \mathbf{T}_{2} \mathbf{y}_{\text {opt }}\right\|_{2}^{2} .
\end{aligned}
$$

Combining all of the above gives

$$
\|\mathbf{B}-\mathbf{A X}\|_{2}^{2} \geq\left\|\left(\mathbf{U T}_{1}+\mathbf{U}_{\perp} \mathbf{T}_{2}\right) \mathbf{y}_{\text {opt }}\right\|_{2}^{2} \geq\left\|\mathbf{U}_{\perp} \mathbf{T}_{2}\right\|_{2}^{2}=\left\|\mathbf{U}_{\perp} \mathbf{U}_{\perp}^{T} \mathbf{B}\right\|_{2}^{2} .
$$

This lower bound is achieved by $\mathbf{X}_{o p t}=\mathbf{A}^{\dagger} \mathbf{B}$,

$$
\left\|\mathbf{B}-\mathbf{A} \mathbf{X}_{\text {opt }}\right\|_{2}^{2}=\left\|\mathbf{B}-\mathbf{A} \mathbf{A}^{\dagger} \mathbf{B}\right\|_{2}^{2}=\left\|\left(\mathbf{I}-\mathbf{U U}^{T}\right) \mathbf{B}\right\|_{2}^{2}=\left\|\mathbf{U}_{\perp} \mathbf{U}_{\perp}^{T} \mathbf{B}\right\|_{2}^{2}
$$

4.2. The Krylov space. The elements of the Krylov space $\mathcal{K}_{q}$ in (2.1) can be expressed in terms of matrices $\hat{\phi}\left(\mathbf{A} \mathbf{A}^{T}\right) \mathbf{A X} \in \mathbb{R}^{m \times s}$, where $\hat{\phi}$ is a polynomial of degree $q$. From the point of view of singular values, though, we need a higher degree polynomial,

$$
\hat{\phi}\left(\mathbf{A} \mathbf{A}^{T}\right) \mathbf{A X}=\mathbf{U} \hat{\phi}\left(\boldsymbol{\Sigma} \boldsymbol{\Sigma}^{T}\right) \boldsymbol{\Sigma} \mathbf{V}^{T} \mathbf{X}=\mathbf{U} \phi(\boldsymbol{\Sigma}) \mathbf{V}^{T} \mathbf{X} .
$$

Here $\phi$ is a polynomial of degree $2 q+1$ with odd powers only, and represents a generalized matrix function [3, 22]. Since

$$
\boldsymbol{\Sigma}=\operatorname{diag}\left(\begin{array}{lll}
\sigma_{1} & \cdots & \sigma_{\min \{m, n\}}
\end{array}\right) \in \mathbb{R}^{m \times n}
$$

is rectangular, the polynomial $\phi$ is applied to the diagonal elements of $\boldsymbol{\Sigma}$ only, and returns a diagonal matrix of the same dimension,

$$
\phi(\boldsymbol{\Sigma}) \equiv \operatorname{diag}\left(\phi\left(\sigma_{1}\right) \quad \cdots \quad \phi\left(\sigma_{\min \{m, n\}}\right)\right) \in \mathbb{R}^{m \times n} .
$$

\footnotetext{
${ }^{4}$ The superscript $\dagger$ denotes the Moore-Penrose inverse.
} 
With this, we denote elements in $\mathcal{K}_{q}$ by

$$
\boldsymbol{\Phi} \equiv \mathbf{U} \phi(\boldsymbol{\Sigma}) \mathbf{V}^{T} \mathbf{X} \in \mathbb{R}^{m \times s} .
$$

Clearly,

$$
\text { range }(\boldsymbol{\Phi}) \subset \mathcal{K}_{q}
$$

The assumption $\operatorname{dim}\left(\mathcal{K}_{q}\right)=(q+1) s \leq m$ from Algorithm 2.1 guarantees that $\mathbf{U}_{K}$ is indeed an orthonormal basis for $\mathcal{K}_{q}$.

4.3. Angles between subspaces. Let $\mathbf{Q} \in \mathbb{R}^{n \times s}$ and $\mathbf{W}_{k} \in \mathbb{R}^{n \times k}$, with $k \leq s$, be matrices with orthonormal columns. Hence, the singular values $\sigma_{j}\left(\mathbf{W}_{k}^{T} \mathbf{Q}\right)$ lie between zero and one, and we can write $\sigma_{j}\left(\mathbf{W}_{k}^{T} \mathbf{Q}\right)=\cos \theta_{j}, 1 \leq j \leq k$. The principal or canonical angles between range $(\mathbf{Q})$ and $\operatorname{range}\left(\mathbf{W}_{k}\right)$ are [19, Section 6.4.3]

$$
0 \leq \theta_{1} \leq \cdots \leq \theta_{k} \leq \pi / 2
$$

where $\theta_{j}=\cos ^{-1}\left(\sigma_{j}\left(\mathbf{W}_{k}^{T} \mathbf{Q}\right)\right)$. Following [41, Definition I.5.3], we define the diagonal matrix of principal angles between the subspaces spanned by the columns of $\mathbf{Q}$ and the columns of $\mathbf{W}_{k}$

$$
\boldsymbol{\Theta}\left(\mathbf{Q}, \mathbf{W}_{k}\right) \equiv \operatorname{diag}\left(\theta_{1} \quad \cdots \quad \theta_{k}\right) .
$$

Hence the singular values of $\mathbf{W}_{k}^{T} \mathbf{Q}$ are the diagonal elements of $\cos \boldsymbol{\Theta}\left(\mathbf{Q}, \mathbf{W}_{k}\right)$. From [19, Section 6.4.3] and [41, Section I.5.3] follows that the distance between range( $\left.\mathbf{W}_{k}\right)$ and range $(\mathbf{Q})$ in the two and Frobenius norms, respectively, equals

$$
\left\|\sin \Theta\left(\mathbf{Q}, \mathbf{W}_{k}\right)\right\|_{2, F}=\left\|\left(\mathbf{I}-\mathbf{W}_{k} \mathbf{W}_{k}^{T}\right) \mathbf{Q}\right\|_{2, F} .
$$

In particular, $\left\|\sin \boldsymbol{\Theta}\left(\mathbf{Q}, \mathbf{W}_{k}\right)\right\|_{2}=\sin \theta_{k}$, so the two norm distance is determined by the largest principal angle.

Assume that range $\left(\mathbf{W}_{k}\right)$ and range $(\mathbf{Q})$ are sufficiently close, so that the largest angle $\theta_{k}<\pi / 2$. This is equivalent to $\cos \boldsymbol{\Theta}\left(\mathbf{Q}, \mathbf{W}_{k}\right)$ being nonsingular, and $\operatorname{rank}\left(\mathbf{W}_{k}^{T} \mathbf{Q}\right)=$ $k$. Then [45, Section 3] implies that the tangents of the principal angles satisfy

$$
\begin{aligned}
\left\|\tan \Theta\left(\mathbf{Q}, \mathbf{W}_{k}\right)\right\|_{2, F} & =\left\|\sin \boldsymbol{\Theta}\left(\mathbf{Q}, \mathbf{W}_{k}\right)\left(\cos \boldsymbol{\Theta}\left(\mathbf{Q}, \mathbf{W}_{k}\right)\right)^{\dagger}\right\|_{2, F} \\
& =\left\|\left(\mathbf{I}-\mathbf{W}_{k} \mathbf{W}_{k}^{T}\right) \mathbf{Q}\left(\mathbf{W}_{k}^{T} \mathbf{Q}\right)^{\dagger}\right\|_{2, F} .
\end{aligned}
$$

As above, $\left\|\tan \boldsymbol{\Theta}\left(\mathbf{Q}, \mathbf{W}_{k}\right)\right\|_{2}=\tan \theta_{k}$, so the two norm tangent is determined by the largest principal angle. The following lemma will be used in subsequent derivations, so we include its simple proof.

LEMma 4.3 (Theorem 3.1 in [45]). Let $\mathbf{Q} \in \mathbb{R}^{n \times s}$ have orthonormal columns, and let $\mathbf{W} \equiv\left(\mathbf{W}_{k} \quad \mathbf{W}_{k, \perp}\right) \in \mathbb{R}^{n \times n}$ be an orthogonal matrix where $\mathbf{W}_{k} \in \mathbb{R}^{n \times k}$ with $k \leq s$. If $\operatorname{rank}\left(\mathbf{W}_{k}^{T} \mathbf{Q}\right)=k$ then

$$
\left\|\tan \boldsymbol{\Theta}\left(\mathbf{Q}, \mathbf{W}_{k}\right)\right\|_{2, F}=\left\|\left(\mathbf{W}_{k, \perp}^{T} \mathbf{Q}\right)\left(\mathbf{W}_{k}^{T} \mathbf{Q}\right)^{\dagger}\right\|_{2, F} .
$$

Proof. From (4.5) and $\mathbf{I}=\mathbf{W}_{k} \mathbf{W}_{k}^{T}+\mathbf{W}_{k, \perp} \mathbf{W}_{k, \perp}^{T}$ follows

$$
\begin{aligned}
\left\|\tan \Theta\left(\mathbf{Q}, \mathbf{W}_{k}\right)\right\|_{2, F} & =\left\|\left(\mathbf{I}-\mathbf{W}_{k} \mathbf{W}_{k}^{T}\right) \mathbf{Q}\left(\mathbf{W}_{k}^{T} \mathbf{Q}\right)^{\dagger}\right\|_{2, F} \\
& =\left\|\mathbf{W}_{k, \perp} \mathbf{W}_{k, \perp}^{T} \mathbf{Q}\left(\mathbf{W}_{k}^{T} \mathbf{Q}\right)^{\dagger}\right\|_{2, F} \\
& =\left\|\mathbf{W}_{k, \perp}^{T} \mathbf{Q}\left(\mathbf{W}_{k}^{T} \mathbf{Q}\right)^{\dagger}\right\|_{2, F} .
\end{aligned}
$$


The last equality follows from the unitary invariance of the two and the Frobenius norms.

4.4. Gap-amplifying and Chebyshev polynomials. We generalize the Chebyshevbased gap-amplifying polynomials in [34, Section 4.4], 43, Section 2.2]. Given an integer $q \geq 1$, define the polynomial

$$
\psi_{q^{\prime}}(x)=\psi_{2 q+1}(x)=\sum_{j=0}^{q} a_{2 j+1} x^{2 j+1}
$$

of degree $q^{\prime}=2 q+1$ with only odd powers of $x$. The polynomial $\psi$ is gap-amplifying if it satisfies three properties:

1. Small input values remain small,

$$
\psi_{q^{\prime}}(1)=1, \quad \text { and } \quad\left|\psi_{q^{\prime}}(x)\right| \leq 1 \quad \text { for } x \in[0,1] .
$$

2. Large input values are amplified,

$$
\psi_{q^{\prime}}(x) \geq x c 2^{q^{\prime} r(x)} \quad \text { for } x \geq 1,
$$

where the constant $c$ and the function $r(x)$ are parameters of $\psi$.

3. Super linear growth for large input values,

$$
\frac{\psi_{q^{\prime}}(x)}{x} \geq \frac{\psi_{q^{\prime}}(y)}{y} \quad \text { for } x \geq y \geq 1 .
$$

The simplest gap-amplifying polynomial is a Chebyshev polynomial.

Lemma 4.4 (Lemma 5 in [34]). The Chebyshev polynomial $T_{q^{\prime}}(x)$ of the first kind contains only odd powers of $x$ and is gap-amplifying with $c=1 / 4$ and $r(x)=$ $\min \{\sqrt{x-1}, 1\}$.

Proof. We give a quick sketch of the proof of [34, Lemma 5]). Clearly, the Chebyshev polynomial $T_{q^{\prime}}(x)$ satisfies Property 1 . To prove Property 3 , it suffices to show

$$
T_{q^{\prime}}^{\prime}(z) \geq \frac{T_{q^{\prime}}(y)}{y} \quad \text { for some } z \geq y \geq 1,
$$

because the mean value theorem implies there exists a $z \in[y, x]$ with

$$
\begin{aligned}
T_{q^{\prime}}(x) & =T_{q^{\prime}}(y+x-y)=T_{q^{\prime}}(y)+T_{q^{\prime}}^{\prime}(z)(x-y) \\
& \geq T_{q^{\prime}}(y)+\frac{T_{q^{\prime}}(y)}{y}(x-y)=x \frac{T_{q^{\prime}}(y)}{y} .
\end{aligned}
$$

Our proof of Property 2 corrects a small typo in a similar proof in 34. Although a bound equivalent to Property 2 is claimed in [34. Lemma 5] it is only proved that $T_{q^{\prime}}(x) \geq c 2^{q^{\prime} r(x)}$. However, only a slight modification is required for the stronger result. The proof of [34, Lemma 5] shows that

$$
T_{q^{\prime}}(x) \geq \frac{1}{2} 2^{q^{\prime} \sqrt{x-1}} \quad \text { for } 1 \leq x \leq 2 .
$$

To derive a lower bound on $T_{q^{\prime}}(x) / x$ for $x \geq 1$, first consider $1 \leq x \leq 2$, where

$$
\frac{T_{q^{\prime}}(x)}{x} \geq \frac{T_{q^{\prime}}(x)}{2} \geq \frac{1}{4} 2^{q^{\prime} \sqrt{x-1}} .
$$


For the remaining case $x>2$, Property 3 implies

$$
\frac{T_{q^{\prime}}(x)}{x} \geq \frac{T_{q^{\prime}}(2)}{2} \geq \frac{1}{4} 2^{q^{\prime}} .
$$

Hence $T_{q^{\prime}}(x) \geq \frac{x}{4} 2^{q^{\prime} \min \{\sqrt{x-1}, 1\}}$.

For our analysis, we use a rescaled version of the gap-amplifying polynomial $T_{q^{\prime}}(x)$, which has similar properties to the original.

LEMMA 4.5. Let

$$
\phi(x)=\frac{(1+\gamma) \alpha}{\psi_{q^{\prime}}(1+\gamma)} \psi_{q^{\prime}}(x / \alpha)
$$

be the rescaled gap-amplifying polynomial. Then

$$
|\phi(x)| \leq \frac{4 \alpha}{2^{q^{\prime} \min \{\sqrt{\gamma}, 1\}}} \quad \text { for } 0 \leq x \leq \alpha .
$$

Proof. The proof is immediate since $\left|\psi_{q^{\prime}}(x / \alpha)\right| \leq 1$ for $0 \leq x \leq \alpha$, and Property 2 implies

$$
\psi_{q^{\prime}}(1+\gamma) \geq \frac{(1+\gamma)}{4} 2^{q^{\prime} \min \{\sqrt{\gamma}, 1\}}
$$

Lemma 4.6. The rescaled gap-amplifying polynomial $\phi(x)$ in (4.7) satisfies

$$
\phi(x) \geq x \quad \text { for } x \geq(1+\gamma) \alpha .
$$

Proof. Property 3 implies

$$
\frac{\psi_{q^{\prime}}(x / \alpha)}{x / \alpha} \geq \frac{\psi_{q^{\prime}}(1+\gamma)}{1+\gamma} \quad \text { for } x \geq(1+\gamma) \alpha .
$$

Now rearrange terms and apply the definition of $\phi(x)$ in (4.7).

4.5. Proof of Lemma 2.4, Let $\phi(x)$ be the rescaled gap-amplifying polynomial in (4.7) with $\alpha=\sigma_{k+1}$ and $\gamma$ in (2.6). The inequalities for $\phi\left(\sigma_{i}\right)$ follow from $q^{\prime}=2 q+1$, Lemma 4.5 and Lemma 4.6,

From $\phi\left(\sigma_{i}\right)>0$ for $1 \leq i \leq k$ and $\phi\left(\boldsymbol{\Sigma}_{k}\right)$ being a diagonal matrix follows

$$
\left\|\phi\left(\boldsymbol{\Sigma}_{k}\right)^{-1}\right\|_{2}=\max _{1 \leq i \leq k} \phi\left(\sigma_{i}\right)^{-1} \leq \max _{1 \leq i \leq k} \sigma_{i}^{-1}=\sigma_{k}^{-1} .
$$

Furthermore

$$
\left\|\phi\left(\boldsymbol{\Sigma}_{k, \perp}\right)\right\|_{2}=\max _{i \geq k+1}\left|\phi\left(\sigma_{i}\right)\right| \leq \frac{4 \sigma_{k+1}}{2^{(2 q+1) \min }\{\sqrt{\gamma}, 1\}} .
$$

5. Proof of Theorem 2.1, for general and orthonormal $\mathrm{X}$. We focus on the case where $\mathbf{X}$ is a general matrix, or has orthonormal columns, and postpone the technicalities required for the full-column rank case to Appendix A.

The first and most critical step of the proof makes a connection between principal angles and least-squares residuals. 
Viewing the sine as a least squares residual. Let $\mathcal{P}_{q}$ be the orthogonal projector onto the Krylov space $\mathcal{K}_{q}$. For $\boldsymbol{\Phi}$ in (4.2) let $\boldsymbol{\Phi} \boldsymbol{\Phi}^{\dagger}$ be the orthogonal projector onto range $(\boldsymbol{\Phi})$, with range $\left(\boldsymbol{\Phi} \boldsymbol{\Phi}^{\dagger}\right) \subset$ range $\left(\mathcal{P}_{q}\right)$ due to (4.3). Hence, (4.4) implies

$$
\left\|\sin \boldsymbol{\Theta}\left(\mathcal{K}_{q}, \mathbf{U}_{k}\right)\right\|_{2, F}=\left\|\left(\mathbf{I}-\mathcal{P}_{q}\right) \mathbf{U}_{k}\right\|_{2, F} \leq\left\|\left(\mathbf{I}-\mathbf{\Phi} \boldsymbol{\Phi}^{\dagger}\right) \mathbf{U}_{k}\right\|_{2, F} .
$$

Lemma 4.2 implies that $\left\|\left(\mathbf{I}-\mathbf{\Phi} \boldsymbol{\Phi}^{\dagger}\right) \mathbf{U}_{k}\right\|_{2, F}$ is the residual of the least squares problem

$$
\left\|\left(\mathbf{I}-\mathbf{\Phi} \boldsymbol{\Phi}^{\dagger}\right) \mathbf{U}_{k}\right\|_{2, F}=\min _{\boldsymbol{\Psi}}\left\|\mathbf{U}_{k}-\boldsymbol{\Phi} \boldsymbol{\Psi}\right\|_{2, F}=\left\|\mathbf{U}_{k}-\boldsymbol{\Phi} \boldsymbol{\Psi}_{\text {opt }}\right\|_{2, F},
$$

where $\boldsymbol{\Psi}_{\text {opt }}=\boldsymbol{\Phi}^{\dagger} \mathbf{U}_{k}$ is a least squares solution.

Focussing on the target space. Decompose $\boldsymbol{\Phi}$ into the target component range $\left(\mathbf{U}_{k}\right)$ and the complementary subspace, $\boldsymbol{\Phi}=\boldsymbol{\Phi}_{k}+\boldsymbol{\Phi}_{k, \perp}$, where

$$
\boldsymbol{\Phi}_{k} \equiv \mathbf{U}_{k} \phi\left(\boldsymbol{\Sigma}_{k}\right) \mathbf{V}_{k}^{T} \mathbf{X}, \quad \boldsymbol{\Phi}_{k, \perp} \equiv \mathbf{U}_{k, \perp} \phi\left(\boldsymbol{\Sigma}_{k, \perp}\right) \mathbf{V}_{k, \perp}^{T} \mathbf{X} .
$$

From $\operatorname{rank}\left(\mathbf{V}_{k}^{T} \mathbf{X}\right)=k$ follows that $\left(\mathbf{V}_{k}^{T} \mathbf{X}\right)^{\dagger}$ is a right inverse, $\left(\mathbf{V}_{k}^{T} \mathbf{X}\right)\left(\mathbf{V}_{k}^{T} \mathbf{X}\right)^{\dagger}=\mathbf{I}_{k}$. With (4.1) this gives

$$
\boldsymbol{\Phi}_{k}^{\dagger}=\left(\mathbf{V}_{k}^{T} \mathbf{X}\right)^{\dagger} \phi\left(\boldsymbol{\Sigma}_{k}\right)^{-1} \mathbf{U}_{k}^{T} \quad \text { and } \quad \boldsymbol{\Phi}_{k} \boldsymbol{\Phi}_{k}^{\dagger}=\mathbf{U}_{k} \mathbf{U}_{k}^{T},
$$

meaning $\boldsymbol{\Phi}_{k} \boldsymbol{\Phi}_{k}^{\dagger}$ is the orthogonal projector onto the target space range $\left(\mathbf{U}_{k}\right)$. The minimality of the least squares residual implies

$$
\begin{aligned}
\left\|\left(\mathbf{I}-\boldsymbol{\Phi} \boldsymbol{\Phi}^{\dagger}\right) \mathbf{U}_{k}\right\|_{2, F} & =\left\|\mathbf{U}_{k}-\boldsymbol{\Phi}\left(\boldsymbol{\Phi}^{\dagger} \mathbf{U}_{k}\right)\right\|_{2, F} \\
& \leq\left\|\mathbf{U}_{k}-\boldsymbol{\Phi}\left(\boldsymbol{\Phi}_{k}^{\dagger} \mathbf{U}_{k}\right)\right\|_{2, F}=\left\|\left(\mathbf{I}-\boldsymbol{\Phi} \boldsymbol{\Phi}_{k}^{\dagger}\right) \mathbf{U}_{k}\right\|_{2, F} .
\end{aligned}
$$

Now replace the other instance of $\boldsymbol{\Phi}$ by (5.2), and use (5.3) to simplify

$$
\begin{aligned}
\left\|\left(\mathbf{I}-\boldsymbol{\Phi}^{\dagger}\right) \mathbf{U}_{k}\right\|_{2, F} & \leq\left\|\left(\mathbf{I}-\boldsymbol{\Phi} \boldsymbol{\Phi}_{k}^{\dagger}\right) \mathbf{U}_{k}\right\|_{2, F}=\left\|\left(\mathbf{I}-\boldsymbol{\Phi}_{k} \boldsymbol{\Phi}_{k}^{\dagger}\right) \mathbf{U}_{k}-\boldsymbol{\Phi}_{k, \perp} \boldsymbol{\Phi}_{k}^{\dagger} \mathbf{U}_{k}\right\|_{2, F} \\
& =\left\|\left(\mathbf{I}-\mathbf{U}_{k} \mathbf{U}_{k}^{T}\right) \mathbf{U}_{k}-\boldsymbol{\Phi}_{k, \perp} \boldsymbol{\Phi}_{k}^{\dagger} \mathbf{U}_{k}\right\|_{2, F} \\
& =\left\|\boldsymbol{\Phi}_{k, \perp} \boldsymbol{\Phi}_{k}^{\dagger} \mathbf{U}_{k}\right\|_{2, F} .
\end{aligned}
$$

Summary so far. Combining (5.1) with (5.4) gives

$$
\left\|\sin \Theta\left(\mathcal{K}_{q}, \mathbf{U}_{k}\right)\right\|_{2, F} \leq\left\|\boldsymbol{\Phi}_{k, \perp} \boldsymbol{\Phi}_{k}^{\dagger} \mathbf{U}_{k}\right\|_{2, F} .
$$

Extracting the polynomials. The expressions for $\boldsymbol{\Phi}_{k, \perp}$ in (5.2) and $\boldsymbol{\Phi}_{k}^{\dagger}$ in (5.3), and submultiplicativity (Section 4.1) yield

$$
\begin{aligned}
\left\|\boldsymbol{\Phi}_{k, \perp} \boldsymbol{\Phi}_{k}^{\dagger} \mathbf{U}_{k}\right\|_{2, F} & =\left\|\mathbf{U}_{k, \perp} \phi\left(\boldsymbol{\Sigma}_{k, \perp}\right) \mathbf{V}_{k, \perp}^{T} \mathbf{X}\left(\mathbf{V}_{k}^{T} \mathbf{X}\right)^{\dagger} \phi\left(\boldsymbol{\Sigma}_{k}\right)^{-1} \mathbf{U}_{k}^{T} \mathbf{U}_{k}\right\|_{2, F} \\
& =\left\|\phi\left(\boldsymbol{\Sigma}_{k, \perp}\right) \mathbf{V}_{k, \perp}^{T} \mathbf{X}\left(\mathbf{V}_{k}^{T} \mathbf{X}\right)^{\dagger} \phi\left(\boldsymbol{\Sigma}_{k}\right)^{-1}\right\|_{2, F} \\
& \leq\left\|\phi\left(\boldsymbol{\Sigma}_{k, \perp}\right)\right\|_{2}\left\|\phi\left(\boldsymbol{\Sigma}_{k}\right)^{-1}\right\|_{2}\left\|\mathbf{V}_{k, \perp}^{T} \mathbf{X}\left(\mathbf{V}_{k}^{T} \mathbf{X}\right)^{\dagger}\right\|_{2, F} .
\end{aligned}
$$

Combining the previous two sets of inequalities gives

$$
\left\|\sin \boldsymbol{\Theta}\left(\mathcal{K}_{q}, \mathbf{U}_{k}\right)\right\|_{2, F} \leq\left\|\phi\left(\boldsymbol{\Sigma}_{k, \perp}\right)\right\|_{2}\left\|\phi\left(\boldsymbol{\Sigma}_{k}\right)^{-1}\right\|_{2}\left\|\mathbf{V}_{k, \perp}^{T} \mathbf{X}\left(\mathbf{V}_{k}^{T} \mathbf{X}\right)^{\dagger}\right\|_{2, F} .
$$

This concludes the proof for general $\mathbf{X}$. The proof for the special case where $\mathbf{X}$ has linearly independent columns follows from Lemma 4.3 
6. Proof of Theorem 2.2, The proof imitates that of Theorem 2.1, and simply substitutes the vectors $\mathbf{u}_{i}$ for the matrix $\mathbf{U}_{k}$. Note that $\left(\mathbf{I}-\mathbf{U}_{k} \mathbf{U}_{k}^{T}\right) \mathbf{u}_{i}=0$ for $1 \leq i \leq k$, which implies

$$
\left|\sin \boldsymbol{\Theta}\left(\mathcal{K}_{q}, \mathbf{u}_{i}\right)\right| \leq\left\|\boldsymbol{\Phi}_{k, \perp} \boldsymbol{\Phi}_{k}^{\dagger} \mathbf{u}_{i}\right\|_{2}, \quad 1 \leq i \leq k .
$$

The expressions for $\boldsymbol{\Phi}_{k, \perp}$ in (5.2) and $\boldsymbol{\Phi}_{k}^{\dagger}$ in (5.3), and submultiplicativity yield

$$
\begin{aligned}
\left\|\boldsymbol{\Phi}_{k, \perp} \boldsymbol{\Phi}_{k}^{\dagger} \mathbf{u}_{k}\right\|_{2} & =\left\|\mathbf{U}_{k, \perp} \phi\left(\boldsymbol{\Sigma}_{k, \perp}\right) \mathbf{V}_{k, \perp}^{T} \mathbf{X}\left(\mathbf{V}_{k}^{T} \mathbf{X}\right)^{\dagger} \phi\left(\boldsymbol{\Sigma}_{k}\right)^{-1} \mathbf{U}_{k}^{T} \mathbf{u}_{i}\right\|_{2} \\
& =\left\|\phi\left(\boldsymbol{\Sigma}_{k, \perp}\right) \mathbf{V}_{k, \perp}^{T} \mathbf{X}\left(\mathbf{V}_{k}^{T} \mathbf{X}\right)^{\dagger} \phi\left(\sigma_{i}\right)^{-1}\right\|_{2} \\
& \leq\left\|\phi\left(\boldsymbol{\Sigma}_{k, \perp}\right)\right\|_{2}\left|\phi\left(\sigma_{i}\right)^{-1}\right|\left\|\mathbf{V}_{k, \perp}^{T} \mathbf{X}\left(\mathbf{V}_{k}^{T} \mathbf{X}\right)^{\dagger}\right\|_{2}
\end{aligned}
$$

Combining the previous two sets of inequalities gives

$$
\left|\sin \boldsymbol{\Theta}\left(\mathcal{K}_{q}, \mathbf{u}_{i}\right)\right| \leq\left\|\phi\left(\boldsymbol{\Sigma}_{k, \perp}\right)\right\|_{2}\left|\phi\left(\sigma_{i}\right)^{-1}\right|\left\|\mathbf{V}_{k, \perp}^{T} \mathbf{X}\left(\mathbf{V}_{k}^{T} \mathbf{X}\right)^{\dagger}\right\|_{2} .
$$

This concludes the proof of the case for general $\mathbf{X}$. The proof for the special case where $\mathbf{X}$ has orthonormal columns follows from Lemma 4.3.

7. Proof of Theorem 2.3. This proof is more involved than the previous ones, and requires two auxiliary results, an alternative expression for the error (Section 7.1), and a bound on its Frobenius norm (Section 7.2).

7.1. An alternative expression for the error. Algorithm 2.1 approximates the dominant left singular vectors of $\mathbf{A}$ by the orthonormal matrix $\hat{\mathbf{U}}_{k} \in \mathbb{R}^{m \times k}$. Since bounding $\left\|\mathbf{A}-\hat{\mathbf{U}}_{k} \hat{\mathbf{U}}_{k}^{T} \mathbf{A}\right\|_{F}$ seems hard, we present an alternative expression that is easier to analyze.

Lemma 7.1 (Lemma 8 in [11). Let $\mathbf{U}_{K}$ be an orthonormal basis for $\mathcal{K}_{q}$ and let $\hat{\mathbf{U}}_{i}$ be as in (2.2), containing the top $i$ columns of the output of Algorithm 2.1. Then

$$
\mathbf{A}-\hat{\mathbf{U}}_{i} \hat{\mathbf{U}}_{i}^{T} \mathbf{A}=\mathbf{A}-\mathbf{U}_{K}\left(\mathbf{U}_{K}^{T} \mathbf{A}\right)_{i}, \quad 1 \leq i \leq k .
$$

In addition, $\mathbf{U}_{K}\left(\mathbf{U}_{K}^{T} \mathbf{A}\right)_{i}$ is a best rank-i approximation to $\mathbf{A}$ from $\mathcal{K}_{q}$ in the Frobenius norm,

$$
\left\|\mathbf{A}-\mathbf{U}_{K}\left(\mathbf{U}_{K}^{T} \mathbf{A}\right)_{i}\right\|_{F}^{2}=\min _{\operatorname{rank}(\mathbf{Y}) \leq i}\left\|\mathbf{A}-\mathbf{U}_{K} \mathbf{Y}\right\|_{F}^{2}, \quad 1 \leq i \leq k .
$$

Proof. Since the transition to best rank- $i$ approximations is a key component, we illustrate how it comes about by proving the first assertion for the case $i=k$.

Algorithm 2.1 outputs $\hat{\mathbf{U}}_{k}=\mathbf{U}_{K} \mathbf{U}_{W, k}$, where $\mathbf{U}_{W, k}$ is the matrix of the dominant $k$ left singular vectors of $\mathbf{W}=\mathbf{U}_{K}^{T} \mathbf{A}$. This means $\mathbf{U}_{W, k}$ spans the same range as $\mathbf{W}_{k}$, the best rank- $k$ approximation to $\mathbf{W}$. Therefore

$$
\begin{aligned}
\mathbf{A}-\hat{\mathbf{U}}_{k} \hat{\mathbf{U}}_{k}^{T} \mathbf{A} & =\mathbf{A}-\mathbf{U}_{K} \mathbf{U}_{W, k} \mathbf{U}_{W, k}^{T} \mathbf{U}_{K}^{T} \mathbf{A} \\
& =\mathbf{A}-\mathbf{U}_{K} \mathbf{W}_{k} \mathbf{W}_{k}^{\dagger} \mathbf{W}=\mathbf{A}-\mathbf{U}_{K} \mathbf{W}_{k} .
\end{aligned}
$$

The last equality follows from $\mathbf{W}_{k} \mathbf{W}_{k}^{\dagger}$ being the orthogonal projector onto range $\left(\mathbf{W}_{k}\right)$. 
Lemma 7.1 shows that (2.3) in Theorem 2.3 can be proved by bounding $\| \mathbf{A}-$ $\mathbf{U}_{K}\left(\mathbf{U}_{K}^{T} \mathbf{A}\right)_{i} \|_{F}$. Next we transition from the best rank- $i$ approximation of the "projected" matrix $\left(\mathbf{U}_{K}^{T} \mathbf{A}\right)_{i}$ to the best rank- $i$ approximation $\mathbf{A}_{i}$ of the original matrix, by splitting for $1 \leq i \leq k$,

$$
\mathbf{A}=\mathbf{A}_{i}+\mathbf{A}_{i, \perp} \quad \text { where } \quad \mathbf{A}_{i}=\mathbf{U}_{i} \boldsymbol{\Sigma}_{i} \mathbf{V}_{i}^{T} \text { and } \mathbf{A}_{i, \perp}=\mathbf{U}_{i, \perp} \boldsymbol{\Sigma}_{i, \perp} \mathbf{V}_{i, \perp}^{T} .
$$

Lemma 7.2. Let $\mathbf{U}_{K}$ be an orthonormal basis for $\mathcal{K}_{q}$, and $\hat{\mathbf{U}}_{i}$ in 2.2) the columns of the output of Algorithm 2.1. Then

$$
\left\|\mathbf{A}-\hat{\mathbf{U}}_{i} \hat{\mathbf{U}}_{i}^{T} \mathbf{A}\right\|_{F}^{2} \leq\left\|\mathbf{A}_{i}-\mathbf{U}_{K} \mathbf{U}_{K}^{T} \mathbf{A}_{i}\right\|_{F}^{2}+\left\|\mathbf{A}_{i, \perp}\right\|_{F}^{2} .
$$

Proof. The optimality of (7.2) in Lemma 7.1 implies

$$
\begin{aligned}
\left\|\mathbf{A}-\hat{\mathbf{U}}_{i} \hat{\mathbf{U}}_{i}^{T} \mathbf{A}\right\|_{F}^{2} & =\left\|\mathbf{A}-\mathbf{U}_{K}\left(\mathbf{U}_{K}^{T} \mathbf{A}\right)_{i}\right\|_{F}^{2} \\
& \leq\left\|\mathbf{A}-\mathbf{U}_{K} \mathbf{U}_{K}^{T} \mathbf{A}_{i}\right\|_{F}^{2} \\
& =\left\|\mathbf{A}_{i}-\mathbf{U}_{K} \mathbf{U}_{K}^{T} \mathbf{A}_{i}\right\|_{F}^{2}+\left\|\mathbf{A}_{i, \perp}\right\|_{F}^{2} .
\end{aligned}
$$

The last equality follows from Lemma 4.1, $\mathrm{c}$

7.2. Bounding the important part of the error. We bound the term in Lemma 7.2 over which we have control, namely $\left\|\mathbf{A}_{i}-\mathbf{U}_{K} \mathbf{U}_{K}^{T} \mathbf{A}_{i}\right\|_{F}^{2}$.

As in Section 5 , let $\mathcal{P}_{q}$ be the orthogonal projector onto $\mathcal{K}_{q}$. For $\boldsymbol{\Phi}$ in (4.2) let $\boldsymbol{\Phi} \boldsymbol{\Phi}^{\dagger}$ be the orthogonal projector onto range $(\boldsymbol{\Phi})$, with $\operatorname{range}\left(\boldsymbol{\Phi} \Phi^{\dagger}\right) \subset \operatorname{range}\left(\mathcal{P}_{q}\right)$ due to (4.3). The leads to the obvious bound

$$
\left\|\mathbf{A}_{i}-\mathbf{U}_{K} \mathbf{U}_{K}^{T} \mathbf{A}_{i}\right\|_{F}=\left\|\mathbf{A}_{i}-\mathcal{P}_{q} \mathbf{A}_{i}\right\|_{F} \leq\left\|\mathbf{A}_{i}-\boldsymbol{\Phi} \boldsymbol{\Phi}^{\dagger} \mathbf{A}_{i}\right\|_{F}, \quad 1 \leq i \leq k .
$$

We don't stop here, though, but go further and pursue a bound in terms of polynomials.

LEMma 7.3. Let $\phi(x)$ be a polynomial of degree $2 q+1$ with odd powers only that satisfies $\phi\left(\sigma_{j}\right) \geq \sigma_{j}$ for $1 \leq j \leq k$. Then

$$
\left\|\mathbf{A}_{i}-\mathbf{U}_{K} \mathbf{U}_{K}^{T} \mathbf{A}_{i}\right\|_{F} \leq\left\|\mathbf{U}_{i} \phi\left(\boldsymbol{\Sigma}_{i}\right)-\boldsymbol{\Phi} \boldsymbol{\Phi}^{\dagger} \mathbf{U}_{i} \phi\left(\boldsymbol{\Sigma}_{i}\right)\right\|_{F}, \quad 1 \leq i \leq k .
$$

Proof. We use the abbreviation $\mathcal{P}_{\phi}^{\perp} \equiv \mathbf{I}-\mathbf{\Phi} \boldsymbol{\Phi}^{\dagger}$, to denote the orthogonal projector onto range $(\boldsymbol{\Phi})^{\perp}$. From (7.3), (17.4) and the unitary invariance of the Frobenius norm follows

$$
\left\|\mathbf{A}_{i}-\mathbf{U}_{K} \mathbf{U}_{K}^{T} \mathbf{A}_{i}\right\|_{F} \leq\left\|\mathcal{P}_{\phi}^{\perp} \mathbf{A}_{i}\right\|_{F}=\left\|\mathcal{P}_{q}^{\perp} \mathbf{U}_{i} \boldsymbol{\Sigma}_{i}\right\|_{F}, \quad 1 \leq i \leq k .
$$

Expressing the squared Frobenius norm as a sum of squared column norms, and then applying the assumption $\sigma_{j} \leq \phi\left(\sigma_{j}\right)$ yields for $1 \leq i \leq k$,

$$
\left\|\mathcal{P}_{\phi}^{\perp} \mathbf{U}_{i} \boldsymbol{\Sigma}_{i}\right\|_{F}^{2}=\sum_{j=1}^{i} \sigma_{j}^{2}\left\|\mathcal{P}_{\phi}^{\perp} \mathbf{u}_{j}\right\|_{2}^{2} \leq \sum_{j=1}^{i} \phi\left(\sigma_{j}\right)^{2}\left\|\mathcal{P}_{\phi}^{\perp} \mathbf{u}_{j}\right\|_{2}^{2}=\left\|\mathcal{P}_{\phi}^{\perp} \mathbf{U}_{i} \phi\left(\boldsymbol{\Sigma}_{i}\right)\right\|_{F}^{2} .
$$


7.3. From projections to least-squares residuals. Now we are ready to apply the approach from Theorem 2.1 and view the result of Lemma 7.3 as a least squares residual.

Lemma 7.4. Under the assumptions of Theorem 2.3.

$$
\left\|\mathbf{U}_{i} \phi\left(\boldsymbol{\Sigma}_{i}\right)-\boldsymbol{\Phi} \boldsymbol{\Phi}^{\dagger} \mathbf{U}_{i} \phi\left(\boldsymbol{\Sigma}_{i}\right)\right\|_{F} \leq\left\|\phi\left(\boldsymbol{\Sigma}_{k, \perp}\right)\right\|_{2}\left\|\mathbf{V}_{k, \perp}^{T} \mathbf{X}\left(\mathbf{V}_{k}^{T} \mathbf{X}\right)^{\dagger}\right\|_{F}
$$

Proof. Based on the orthogonality $\left(\mathbf{I}-\mathbf{U}_{k} \mathbf{U}_{k}^{T}\right) \mathbf{U}_{i}=\mathbf{0}$ for $1 \leq i \leq k$, we can deduce as in (5.4) that

$$
\left\|\left(\mathbf{I}-\boldsymbol{\Phi} \boldsymbol{\Phi}^{\dagger}\right) \mathbf{U}_{i} \phi\left(\boldsymbol{\Sigma}_{i}\right)\right\|_{F} \leq\left\|\boldsymbol{\Phi}_{k, \perp} \boldsymbol{\Phi}_{k}^{\dagger} \mathbf{U}_{i} \phi\left(\boldsymbol{\Sigma}_{i}\right)\right\|_{F} .
$$

The expressions for $\boldsymbol{\Phi}_{k, \perp}$ in (5.2) and $\boldsymbol{\Phi}_{k}^{\dagger}$ in (5.3), along with the strong submultiplicativity in Section 4.1 yield

$$
\begin{aligned}
\left\|\boldsymbol{\Phi}_{k, \perp} \boldsymbol{\Phi}_{k}^{\dagger} \mathbf{U}_{i} \phi\left(\boldsymbol{\Sigma}_{i}\right)\right\|_{F} & =\left\|\mathbf{U}_{k, \perp} \phi\left(\boldsymbol{\Sigma}_{k, \perp}\right) \mathbf{V}_{k, \perp}^{T} \mathbf{X}\left(\mathbf{V}_{k}^{T} \mathbf{X}\right)^{\dagger} \phi\left(\boldsymbol{\Sigma}_{k}\right)^{-1} \mathbf{U}_{k}^{T} \mathbf{U}_{i} \phi\left(\boldsymbol{\Sigma}_{i}\right)\right\|_{F} \\
& \leq\left\|\phi\left(\boldsymbol{\Sigma}_{k, \perp}\right)\right\|_{2}\left\|\mathbf{V}_{k, \perp}^{T} \mathbf{X}\left(\mathbf{V}_{k}^{T} \mathbf{X}\right)^{\dagger}\right\|_{F} .
\end{aligned}
$$

The above inequality is obtained by noting that for $i=k$ we have $\phi\left(\boldsymbol{\Sigma}_{k}\right)^{-1} \mathbf{U}_{k}^{T} \mathbf{U}_{i} \phi\left(\boldsymbol{\Sigma}_{i}\right)=$ $\mathbf{I}_{k}$, while for $1 \leq i<k$,

$$
\phi\left(\boldsymbol{\Sigma}_{k}\right)^{-1} \mathbf{U}_{k}^{T} \mathbf{U}_{i} \phi\left(\boldsymbol{\Sigma}_{i}\right)=\left(\begin{array}{c}
\mathbf{I}_{i} \\
\mathbf{0}_{(k-i) \times i}
\end{array}\right) .
$$

7.4. Concluding the proof of Theorem 2.3. We prove each of the three inequalities in turn. Recall that

$$
\Delta \equiv\left\|\phi\left(\boldsymbol{\Sigma}_{k, \perp}\right)\right\|_{2}\left\|\mathbf{V}_{k, \perp}^{T} \mathbf{X}\left(\mathbf{V}_{k}^{T} \mathbf{X}\right)^{\dagger}\right\|_{F} .
$$

Proof of (2.3). Combining Lemmas 7.2 [7.3, and 7.4 and recognizing the expression for $\Delta$ yields

$$
\begin{aligned}
\left\|\mathbf{A}-\hat{\mathbf{U}}_{i} \hat{\mathbf{U}}_{i}^{T}\right\|_{F}^{2} & \leq\left\|\mathbf{A}_{i, \perp}\right\|_{F}^{2}+\left\|\phi\left(\boldsymbol{\Sigma}_{k, \perp}\right)\right\|_{2}^{2}\left\|\mathbf{V}_{k, \perp}^{T} \mathbf{X}\left(\mathbf{V}_{k}^{T} \mathbf{X}\right)^{\dagger}\right\|_{F}^{2}, \\
& =\left\|\mathbf{A}_{i, \perp}\right\|_{F}^{2}+\Delta^{2}, \quad 1 \leq i \leq k .
\end{aligned}
$$

Inserting $\left\|\mathbf{A}_{i, \perp}\right\|_{F}=\left\|\mathbf{A}-\mathbf{A}_{i}\right\|_{F}$ gives

$$
\left\|\mathbf{A}-\hat{\mathbf{U}}_{i} \hat{\mathbf{U}}_{i}^{T} \mathbf{A}\right\|_{F}^{2} \leq\left\|\mathbf{A}-\mathbf{A}_{i}\right\|_{F}^{2}+\Delta^{2}, \quad 1 \leq i \leq k .
$$

Taking advantage of the inequality below for scalars $\alpha, \beta \geq 0$,

$$
\sqrt{\alpha^{2}+\beta^{2}} \leq \sqrt{\alpha^{2}+\beta^{2}+2 \alpha \beta}=\sqrt{(\alpha+\beta)^{2}}=\alpha+\beta,
$$

gives the weaker, but square-free bound (2.3).

Proof of (2.4). We use [20, Theorem 3.4], which shows that an additive error bound for a low-rank approximation in the Frobenius norm implies the same in the two norm.

Lemma 7.5 (Theorem 3.4 in $[20]$ ). Given $\mathbf{A}, \tilde{\mathbf{A}} \in \mathbb{R}^{m \times n}$ with $\operatorname{rank}(\tilde{\mathbf{A}})=k<$ $\operatorname{rank}(\mathbf{A})$. If $\|\mathbf{A}-\tilde{\mathbf{A}}\|_{F}^{2} \leq\left\|\mathbf{A}-\mathbf{A}_{k}\right\|_{F}^{2}+\delta$, then

$$
\|\mathbf{A}-\tilde{\mathbf{A}}\|_{2}^{2} \leq\left\|\mathbf{A}-\mathbf{A}_{k}\right\|_{2}^{2}+\delta .
$$

Apply Lemma 7.5 to (7.6), to get $\left\|\mathbf{A}-\hat{\mathbf{U}}_{i} \hat{\mathbf{U}}_{i}^{T} \mathbf{A}\right\|_{2}^{2} \leq\left\|\mathbf{A}-\mathbf{A}_{i}\right\|_{2}^{2}+\Delta^{2}$, and take square roots based on (7.7). 
Proof of (2.5). The upper bounds follow from the minimax theorem for singular values [19, Theorem 8.6.1].

This leaves the lower bounds. Recall the non-increasing ordering of the singular values $\sigma_{1} \geq \cdots \geq \sigma_{k}$, and the fact that $\hat{\mathbf{U}}_{i}$ in (2.2) has orthonormal columns.

Case $i=1$. Apply Lemma 4.1 to (7.5)

$$
\|\mathbf{A}\|_{F}^{2}-\left\|\hat{\mathbf{u}}_{1}^{T} \mathbf{A}\right\|_{F}^{2}=\left\|\mathbf{A}-\hat{\mathbf{u}}_{1} \hat{\mathbf{u}}_{1}^{T} \mathbf{A}\right\|_{F}^{2} \leq\left\|\mathbf{A}_{1, \perp}\right\|_{F}^{2}+\Delta^{2} .
$$

From $\|\mathbf{A}\|_{F}^{2}-\left\|\mathbf{A}_{1, \perp}\right\|_{F}^{2}=\sigma_{1}^{2}$ follows $\sigma_{1}^{2} \leq\left\|\mathbf{A}-\hat{\mathbf{u}}_{1} \hat{\mathbf{u}}_{1}^{T} \mathbf{A}\right\|_{F}^{2}+\Delta^{2}$. Taking square roots based on (7.7) proves (2.5) for $i=1$.

Case $2 \leq i \leq k$. Among all matrices of rank $i-1$, the matrix $\mathbf{A}_{i-1}$ is closest to $\mathbf{A}$ in the Frobenius norm. Hence

$$
\left\|\mathbf{A}_{i-1, \perp}\right\|_{F}=\left\|\mathbf{A}-\mathbf{A}_{i-1}\right\|_{F} \leq\left\|\mathbf{A}-\hat{\mathbf{U}}_{i-1} \hat{\mathbf{U}}_{i-1}^{T} \mathbf{A}\right\|_{F} .
$$

The above, together with the outer product representation $\hat{\mathbf{U}}_{i} \hat{\mathbf{U}}_{i}^{T}=\hat{\mathbf{U}}_{i-1} \hat{\mathbf{U}}_{i-1}^{T}+\hat{\mathbf{u}}_{i} \hat{\mathbf{u}}_{i}^{T}$, Lemma 4.1 and (7.5) gives

$$
\begin{aligned}
\left\|\mathbf{A}_{i-1, \perp}\right\|_{F}^{2}-\left\|\hat{\mathbf{u}}_{i} \hat{\mathbf{u}}_{i}^{T} \mathbf{A}\right\|_{F}^{2} & \leq\left\|\mathbf{A}-\hat{\mathbf{U}}_{i-1} \hat{\mathbf{U}}_{i-1}^{T} \mathbf{A}\right\|_{F}^{2}-\left\|\hat{\mathbf{u}}_{i} \hat{\mathbf{u}}_{i}^{T} \mathbf{A}\right\|_{F}^{2} \\
& =\left\|\mathbf{A}-\hat{\mathbf{U}}_{i} \hat{\mathbf{U}}_{i}^{T} \mathbf{A}\right\|_{F}^{2} \leq\left\|\mathbf{A}_{i, \perp}\right\|_{F}^{2}+\Delta^{2} .
\end{aligned}
$$

At last, applying $\left\|\mathbf{A}_{i-1, \perp}\right\|_{F}^{2}-\left\|\mathbf{A}_{i, \perp}\right\|_{F}^{2}=\sigma_{i}^{2}$, and taking square roots based on (7.7) proves (2.5) for $2 \leq i \leq k$.

This concludes the proof for general $\mathbf{X}$. The proof for the special case where $\mathbf{X}$ has orthonormal columns follows from Lemma 4.3.

8. Conclusions and open problems. Motivated by the emergence of randomized Krylov space methods for low-rank approximations [34, 43, we presented a "proof of concept", that is, structural results for the accuracy of approximate dominant subspaces.

Several open problems arise from our work:

1. Can we better understand and close the disconnect between low-rank approximations and dominant subspace computations?

A singular value gap is a must for dominant subspace computations, if only to ensure wellposedness of the mathematical problem. In contrast, low-rank approximations can do without a gap for special starting guesses $\mathbf{X}$ 34. This comes at the detriment of accuracy, though. Bolstered by a gap, subspace accuracy exhibits the logarithmic dependence (2.7) on $\epsilon$, while, without a gap, the accuracy of a low-rank approximation has only polynomial dependence on $\epsilon$. To the best of our knowledge, gap-independent results are not known for arbitrary $\mathbf{X}$. As the analysis [34] only exploits the fact that $\mathbf{X}$ can give an approximation that is polynomially close to optimal in the Frobenius norm, it could potentially be extended to a variety of random starting guesses.

2. Is it possible to relax the full-rank assumption for $\mathbf{V}_{k}^{T} \mathbf{X}$ ?

Our proofs require $\operatorname{rank}\left(\mathbf{V}_{k}^{T} \mathbf{X}\right)=k$, which forces starting guesses to have at least $s \geq k$ columns. Thus, even in the presence of the requisite singular value gaps, our proofs collapse for starting guesses that consist of a single column.

3. Are our bounds tight enough to be informative, and how relevant are they for practical numerical implementations of block Krylov methods?

9. Acknowledgments. We thank Mark Embree for many useful discussions.

Appendix A. More general proof of Theorem 2.1. The proof below applies to starting guesses $\mathbf{X}$ with linearly independent columns and consists of several steps. 
Preparing $\mathbf{X}$. Since subspace angles are defined by matrices with orthonormal columns, we perform a thin $\mathrm{QR}$ decomposition $\mathbf{X}=\mathbf{Q R}$, where $\mathbf{Q} \in \mathbb{R}^{n \times s}$ has orthonormal columns, and $\mathbf{R} \in \mathbb{R}^{s \times s}$ is nonsingular. Then $\operatorname{range}(\mathbf{Q})=\operatorname{range}(\mathbf{X})$.

The expression for $\mathbf{\Phi}$ contains a basis transformation on $\mathbf{X}$ with the orthogonal matrix $\mathbf{V}$, resulting in a $n \times s$ matrix

$$
\mathbf{V}^{T} \mathbf{Q}=\left(\begin{array}{c}
\mathbf{V}_{k}^{T} \mathbf{Q} \\
\mathbf{V}_{k, \perp}^{T} \mathbf{Q}
\end{array}\right)=\left(\begin{array}{c}
\mathbf{Q}_{k} \\
\mathbf{Q}_{k, \perp}
\end{array}\right)
$$

with orthonormal columns. It remains to account for $\mathbf{R}$ :

$$
\mathbf{V}^{T} \mathbf{X}=\left(\mathbf{V}^{T} \mathbf{Q}\right) \mathbf{R}=\left(\begin{array}{c}
\mathbf{Q}_{k}^{T} \mathbf{R} \\
\mathbf{Q}_{k, \perp}^{T} \mathbf{R}
\end{array}\right)=\left(\begin{array}{c}
\mathbf{X}_{k} \\
\mathbf{X}_{k, \perp}
\end{array}\right)
$$

By assumption, $k=\operatorname{rank}\left(\mathbf{V}_{k}^{T} \mathbf{Q}\right)=\operatorname{rank}\left(\mathbf{X}_{k}\right)=\operatorname{rank}\left(\mathbf{Q}_{k}\right)$, so that $\mathbf{X}_{k} \in \mathbb{R}^{k \times s}$ and $\mathbf{Q}_{k} \in \mathbb{R}^{k \times S}$ have full row rank. In particular,

$$
\mathbf{Q}_{k} \mathbf{Q}_{k}^{\dagger}=\mathbf{I}_{k}, \quad \mathbf{Q}_{k}^{\dagger}=\mathbf{Q}_{k}^{T}\left(\mathbf{Q}_{k} \mathbf{Q}_{k}^{T}\right)^{-1} .
$$

For $\mathbf{X}_{k}$, though, we forego the Moore-Penrose inverse, and choose instead a $(1,2,3)$ inverse [12, Definition 6.2.4]. The matrix

$$
\mathbf{X}_{k}^{+} \equiv \mathbf{R}^{-1} \mathbf{Q}_{k}^{\dagger}
$$

is a right inverse, $\mathbf{X}_{k} \mathbf{X}_{k}^{+}=\mathbf{I}_{k}$, and satisfies three of the four Moore-Penrose conditions,

$$
\mathbf{X}_{k} \mathbf{X}_{k}^{+} \mathbf{X}_{k}=\mathbf{X}_{k}, \quad \mathbf{X}_{k}^{+} \mathbf{X}_{k} \mathbf{X}_{k}^{+}=\mathbf{X}_{k}^{+}, \quad\left(\mathbf{X}_{k} \mathbf{X}_{k}^{+}\right)^{T}=\left(\mathbf{X}_{k} \mathbf{X}_{k}^{+}\right) .
$$

Viewing the sine as a least squares residual. Let $\mathcal{P}_{q}$ be the orthogonal projector onto the Krylov space $\mathcal{K}_{q}$, and $\boldsymbol{\Phi} \boldsymbol{\Phi}^{\dagger}$ the orthogonal projector onto range $(\boldsymbol{\Phi})$. From range $(\boldsymbol{\Phi}) \subset \mathcal{K}_{q}$ follows range $\left(\boldsymbol{\Phi} \Phi^{\dagger}\right) \subset \operatorname{range}\left(\mathcal{P}_{q}\right)$, hence (4.4) implies

$$
\begin{aligned}
\left\|\sin \Theta\left(\mathcal{K}_{q}, \mathbf{U}_{k}\right)\right\|_{2, F} & =\left\|\left(\mathbf{I}-\mathcal{P}_{q}\right) \mathbf{U}_{k}\right\|_{2, F}\left\|\left(\mathbf{I}-\mathbf{\Phi} \boldsymbol{\Phi}^{\dagger}\right) \mathbf{U}_{k}\right\|_{2, F} \\
& =\left\|\sin \boldsymbol{\Theta}\left(\mathbf{\Phi}, \mathbf{U}_{k}\right)\right\|_{2, F} .
\end{aligned}
$$

Lemma 4.2 shows that $\left\|\left(\mathbf{I}-\mathbf{\Phi} \boldsymbol{\Phi}^{\dagger}\right) \mathbf{U}_{k}\right\|_{2, F}$ is the residual of the least squares problem

$$
\left\|\left(\mathbf{I}-\mathbf{\Phi} \boldsymbol{\Phi}^{\dagger}\right) \mathbf{U}_{k}\right\|_{2, F}=\min _{\Psi}\left\|\mathbf{U}_{k}-\boldsymbol{\Phi} \boldsymbol{\Psi}\right\|_{2, F}=\left\|\mathbf{U}_{k}-\mathbf{\Phi} \boldsymbol{\Psi}_{\text {opt }}\right\|_{2, F},
$$

where $\boldsymbol{\Psi}_{\text {opt }}=\boldsymbol{\Phi}^{\dagger} \mathbf{U}_{k}$ is a least squares solution.

Focussing on the target space. Decompose $\boldsymbol{\Phi}$ into the target component range $\left(\mathbf{U}_{k}\right)$ and the complementary subspace, $\boldsymbol{\Phi}=\boldsymbol{\Phi}_{k}+\boldsymbol{\Phi}_{k, \perp}$, where

$$
\boldsymbol{\Phi}_{k} \equiv \mathbf{U}_{k} \phi\left(\boldsymbol{\Sigma}_{k}\right) \mathbf{X}_{k}, \quad \boldsymbol{\Phi}_{k, \perp} \equiv \mathbf{U}_{k, \perp} \phi\left(\boldsymbol{\Sigma}_{k, \perp}\right) \mathbf{X}_{k, \perp}
$$

It is easy to verify that

$$
\boldsymbol{\Phi}_{k}^{+} \equiv \mathbf{X}_{k}^{+} \phi\left(\boldsymbol{\Sigma}_{k}\right)^{-1} \mathbf{U}_{k}^{T}
$$

satisfies the conditions of a $(1,2,3)$ inverse. The minimality of the least squares residual implies

$$
\begin{aligned}
\left\|\left(\mathbf{I}-\mathbf{\Phi} \boldsymbol{\Phi}^{\dagger}\right) \mathbf{U}_{k}\right\|_{2, F} & =\left\|\mathbf{U}_{k}-\boldsymbol{\Phi}\left(\boldsymbol{\Phi}^{\dagger} \mathbf{U}_{k}\right)\right\|_{2, F} \\
& \leq\left\|\mathbf{U}_{k}-\boldsymbol{\Phi}\left(\boldsymbol{\Phi}_{k}^{+} \mathbf{U}_{k}\right)\right\|_{2, F}=\left\|\left(\mathbf{I}-\mathbf{\Phi} \boldsymbol{\Phi}_{k}^{+}\right) \mathbf{U}_{k}\right\|_{2, F} .
\end{aligned}
$$


Now replace the other instance of $\boldsymbol{\Phi}$ by (A.3),

$$
\begin{aligned}
\left\|\left(\mathbf{I}-\boldsymbol{\Phi} \boldsymbol{\Phi}^{+}\right) \mathbf{U}_{k}\right\|_{2, F} & \leq\left\|\left(\mathbf{I}-\boldsymbol{\Phi} \boldsymbol{\Phi}_{k}^{+}\right) \mathbf{U}_{k}\right\|_{2, F} \\
& =\left\|\left(\mathbf{I}-\boldsymbol{\Phi}_{k} \boldsymbol{\Phi}_{k}^{+}\right) \mathbf{U}_{k}-\boldsymbol{\Phi}_{k, \perp} \boldsymbol{\Phi}_{k}^{+} \mathbf{U}_{k}\right\|_{2, F} .
\end{aligned}
$$

Since a $(1,2,3)$ inverse is an orthogonal projector [8, (2.2.13) in Section 2.2.1], it follows that $\boldsymbol{\Phi}_{k} \boldsymbol{\Phi}_{k}^{+}=\mathbf{U}_{k} \mathbf{U}_{k}^{T}$ is the orthogonal projector onto the target space range $\left(\mathbf{U}_{k}\right)$. This observation simplifies A.5,

$$
\begin{aligned}
\left\|\left(\mathbf{I}-\boldsymbol{\Phi}_{k} \boldsymbol{\Phi}_{k}^{+}\right) \mathbf{U}_{k}-\boldsymbol{\Phi}_{k, \perp} \boldsymbol{\Phi}_{k}^{+} \mathbf{U}_{k}\right\|_{2, F} & =\left\|\left(\mathbf{I}-\mathbf{U}_{k} \mathbf{U}_{k}^{T}\right) \mathbf{U}_{k}-\boldsymbol{\Phi}_{k, \perp} \boldsymbol{\Phi}_{k}^{+} \mathbf{U}_{k}\right\|_{2, F} \\
& =\left\|\boldsymbol{\Phi}_{k, \perp} \boldsymbol{\Phi}_{k}^{+} \mathbf{U}_{k}\right\|_{2, F} .
\end{aligned}
$$

Summary so far. Combining the above with (A.2) and (A.5) gives

$$
\left\|\sin \boldsymbol{\Theta}\left(\mathcal{K}_{q}, \mathbf{U}_{k}\right)\right\|_{2, F} \leq\left\|\sin \boldsymbol{\Theta}\left(\boldsymbol{\Phi}, \mathbf{U}_{k}\right)\right\|_{2, F} \leq\left\|\boldsymbol{\Phi}_{k, \perp} \boldsymbol{\Phi}_{k}^{+} \mathbf{U}_{k}\right\|_{2, F} .
$$

Extracting the polynomials. The expressions for $\boldsymbol{\Phi}_{k, \perp}$ in A.3 and $\boldsymbol{\Phi}_{k}^{+}$in (A.4), and submultiplicativity (Section 4.1) yield

$$
\begin{aligned}
\left\|\boldsymbol{\Phi}_{k, \perp} \boldsymbol{\Phi}_{k}^{+} \mathbf{U}_{k}\right\|_{2, F} & =\left\|\phi\left(\boldsymbol{\Sigma}_{k, \perp}\right) \mathbf{X}_{k, \perp} \mathbf{X}_{k}^{+} \phi\left(\boldsymbol{\Sigma}_{k}\right)^{-1}\right\|_{2, F} \\
& \leq\left\|\phi\left(\boldsymbol{\Sigma}_{k, \perp}\right)\right\|_{2}\left\|\phi\left(\boldsymbol{\Sigma}_{k}\right)^{-1}\right\|_{2}\left\|\mathbf{X}_{k, \perp} \mathbf{X}_{k}^{+}\right\|_{2, F} .
\end{aligned}
$$

Combining the previous two sets of inequalities gives

$$
\left\|\sin \boldsymbol{\Theta}\left(\mathcal{K}_{q}, \mathbf{U}_{k}\right)\right\|_{2, F} \leq\left\|\phi\left(\boldsymbol{\Sigma}_{k, \perp}\right)\right\|_{2}\left\|\phi\left(\boldsymbol{\Sigma}_{k}\right)^{-1}\right\|_{2}\left\|\mathbf{X}_{k, \perp} \mathbf{X}_{k}^{+}\right\|_{2, F} .
$$

We chose the $(1,2,3)$ inverse so that $\mathbf{R}$ cancels out, $\left\|\mathbf{X}_{k, \perp} \mathbf{X}_{k}^{+}\right\|_{2, F}=\left\|\mathbf{Q}_{k, \perp} \mathbf{Q}_{k}^{\dagger}\right\|_{2, F}$, and

$$
\left\|\sin \boldsymbol{\Theta}\left(\mathcal{K}_{q}, \mathbf{U}_{k}\right)\right\|_{2, F} \leq\left\|\phi\left(\boldsymbol{\Sigma}_{k, \perp}\right)\right\|_{2}\left\|\phi\left(\boldsymbol{\Sigma}_{k}\right)^{-1}\right\|_{2}\left\|\mathbf{Q}_{k, \perp} \mathbf{Q}_{k}^{\dagger}\right\|_{2, F} .
$$

At last, Lemma 4.3 and range $(\mathbf{Q})=\operatorname{range}(\mathbf{X})$ imply

$$
\left\|\mathbf{Q}_{k, \perp} \mathbf{Q}_{k}^{\dagger}\right\|_{2, F}=\left\|\tan \boldsymbol{\Theta}\left(\mathbf{Q}, \mathbf{V}_{k}\right)\right\|_{2, F}=\left\|\tan \boldsymbol{\Theta}\left(\mathbf{X}, \mathbf{V}_{k}\right)\right\|_{2, F} .
$$

\section{REFERENCES}

[1] D. Achlioptas, Database-friendly random projections, in Proceedings of the twentieth ACM SIGMOD-SIGACT-SIGART symposium on Principles of database systems - PODS '01, New York, New York, USA, May 2001, ACM Press, pp. 274-281.

[2] N. Ailon and B. Chazelle, The fast Johnson Lindenstrauss transform and approximate nearest neighbors, SIAM J. Comput., 39 (2009), pp. 302-322.

[3] F. Arrigo, M. Benzi, and C. Fenu, Computation of generalized matrix functions, SiAM J. Matrix Anal. Appl., 37 (2016), pp. 836-860.

[4] J. Baglama and L. Reichel, Augmented implicitly restarted Lanczos bidiagonalization methods, SIAM J. Sci. Comput., 27 (2005), pp. 19-42.

[5] —_, Restarted block Lanczos bidiagonalization methods, Numer. Alg., 43 (2006), pp. 251272.

[6] C. Beattie, M. Embree, And J. Rossi, Convergence of restarted Krylov subspaces to invariant subspaces, SIAM J. Matrix Anal. Appl., 25 (2004), pp. 1074-1109.

[7] C. Beattie, M. Embree, And D. C. Sorensen, Convergence of polynomial restart Krylov methods for eigenvalue computations, SIAM Rev., 47 (2005), pp. 492-515.

[8] A. BJörCK, Numerical Methods in Matrix Computations, Springer, Heidelberg, 2015.

[9] N. Bosner And Z. Drmač, Subspace gap residuals for Rayleigh-Ritz approximations, SIAM J. Matrix Anal. Appl., 31 (2009), pp. 54-67. 
[10] C. Boutsidis, P. Drineas, And M. Magdon-Ismail, Near-optimal column-based matrix reconstruction, in 2011 IEEE 52nd Annual Symposium on Foundations of Computer ScienceFOCS 2011, IEEE Computer Soc., Los Alamitos, CA, 2011, pp. 305-314.

[11] — Near-optimal column-based matrix reconstruction, SIAM J. Comput., 43 (2014), pp. 687-717.

[12] S. L. Campbell and C. D. Meyer, Generalized Inverses of Linear Transformations, Dover, New York, 1991.

[13] K. L. Clarkson And D. P. WoodrufF, Low rank approximation and regression in input sparsity time, in Proceedings of the 45th annual ACM symposium on Symposium on Theory of Computing (STOC), ACM Press, 2013, p. 81.

[14] J. D. DIXON, Estimating extreme eigenvalues and condition numbers of matrices, SIAM J. Numer. Anal., 20 (1983), pp. 812-814.

[15] P. Drineas and M. W. Mahoney, RandNLA: Randomized Numerical Linear Algebra, Commun. ACM, 59 (2016), pp. 80-90.

[16] P. Drineas, M. W. Mahoney, and S. Muthukrishnan, Relative error CUR matrix decompositions, SIAM J. Matrix Anal. Appl., 30 (2008), pp. 844-881.

[17] P. Drineas, M. W. Mahoney, S. Muthukrishnan, and T. Sarlós, Faster least squares approximation, Numer. Math., 117 (2011), pp. 219-249.

[18] Z. DRMAC̆, On relative residual bounds for the eigenvalues of a Hermitian matrix, Linear Algebra Appl., 244 (1996), pp. 155-163.

[19] G. H. Golub and C. F. Van Loan, Matrix Computations, The Johns Hopkins University Press, Baltimore, fourth ed., 2013.

[20] M. Gu, Subspace iteration randomization and singular value problems, SIAM J. Sci. Comput., 37 (2015), pp. A1139-A1173.

[21] N. Halko, P.-G. Martinsson, and J. A. Tropp, Finding structure with randomness: Probabilistic algorithms for constructing approximate matrix decompositions, SIAM Rev., 53 (2011), pp. 217-288.

[22] J. B. HaWkins And A. Ben-Israel, On generalized matrix functions, Linear Multilinear Algebra, 1 (1973), pp. 163-171.

[23] R. A. Horn and C. R. Johnson, Topics in Matrix Analysis, Cambridge University Press, Cambridge, 1991.

[24] E. R. Jessup And I. C. F. Ipsen, Improving the accuracy of inverse iteration, SIAM J. Sci. Stat. Comput., 13 (1992), pp. 550-72.

[25] Z. JiA, The regularization theory of the Krylov iterative solvers $L S Q R$ and CGLS for linear discrete ill-posed problems, part I: the simple singular value case, arXiv:1701.05708v1 [math.NA], (2017), pp. 1-49.

[26] A. V. KNYAZEv, Convergence rate estimates for iterative methods for a mesh symmetric eigenvalue problem, Soviet J. Numer. Anal. Math. Modelling, 2 (1987), pp. 371-396. Translated from the Russian.

[27] J. KuCZYŃSKI AND H. WoŹNIAKOWSKI, Estimating the largest eigenvalue by the power and Lanczos algorithms with a random start, SIAM J. Matrix Anal. Appl., 13 (1992), pp. 10941122 .

[28] - Probabilistic bounds on the extremal eigenvalues and condition number by the Lanczos algorithm, SIAM J. Matrix Anal. Appl., 15 (1994), pp. 672-691.

[29] R.-C. Li AND L.-H. ZhANG, Convergence of the block Lanczos method for eigenvalue clusters, Numer. Math., 131 (2015), pp. 83-113.

[30] A. Magen And A. Zouzias, Low rank matrix-valued chernoff bounds and approximate matrix multiplication, in Proceedings of the twenty-second annual ACM-SIAM symposium on Discrete Algorithms (SODA), Society for Industrial and Applied Mathematics, Jan 2011, pp. $1422-1436$.

[31] P. J. MAHER, Some norm inequalities concerning generalized inverses, Linear Algebra Appl., 174 (1992), pp. 99-110.

[32] M. Mahoney and P. Drineas, Structural properties underlying high-quality randomized numerical linear algebra algorithms, in Handbook of Big Data, 2016, pp. 137-154.

[33] X. Meng and M. W. Mahoney, Low-distortion Subspace Embeddings in Input-sparsity Time and Applications to Robust Linear Regression, in Proceedings of the 45th annual ACM symposium on Symposium on theory of computing - STOC '13, 2013, p. 91.

[34] C. Musco And C. Musco, Randomized block krylov methods for stronger and faster approximate singular value decomposition, in Advances in Neural Information Processing Systems 28, C. Cortes, N. D. Lawrence, D. D. Lee, M. Sugiyama, and R. Garnett, eds., Curran Associates, Inc., 2015, pp. 1396-1404.

[35] J. Nelson And H. L. NGUYen, OSNAP: Faster numerical linear algebra algorithms via sparser 
subspace embeddings, in Proceedings of the Annual IEEE Symposium on Foundations of Computer Science (FOCS), 2013.

[36] N. H. Nguyen, T. T. Do, And T. D. Tran, A fast and efficient algorithm for low-rank approximation of a matrix, in Proceedings of the 41st annual ACM symposium on Symposium on theory of computing - STOC '09, New York, New York, USA, May 2009, ACM Press, p. 215.

[37] Y. SAAD, On the rates of convergence of the Lanczos and the block-Lanczos methods, SIAM J. Numer. Anal., 17 (1980), pp. 687-706.

[38] — Numerical Methods for Large Eigenvalue Problems, Classics in Applied Mathematics, SIAM, Philadelphia, revised ed., 2011.

[39] T. SARLós, Improved Approximation Algorithms for Large Matrices via Random Projections, in 47th Annual IEEE Symposium on Foundations of Computer Science (FOCS'06), IEEE, Oct 2006, pp. 143-152.

[40] H. D. Simon AND H. ZHA, Low-rank matrix approximation using the Lanczos bidiagonalization process with applications, SIAM J. Sci. Comput., 21 (2000), pp. 2257-2274.

[41] G. W. Stewart and J. Sun, Matrix Perturbation Theory, Academic Press, San Diego, 1990.

[42] J. A. Tropp, Improved analysis of the subsampled randomized Hadamard transform, Advances in Adaptive Data Analysis, 03 (2010), p. 8.

[43] S. WANG, Z. Zhang, AND T. ZhANG, Improved analyses of the randomized power method and block Lanczos method, arXiv:1508.06429v2 [cs.NA], (2015), pp. 1-22.

[44] D. P. Woodruff, Sketching as a tool for numerical linear algebra, Found. Trends Theor. Comput. Sci., 10 (2014), pp. 1-157.

[45] P. Zhu And A. V. Knyazev, Angles between subspaces and their tangents, J. Numer. Math., 21 (2013), pp. 325-340. 